\title{
THE UNUSUALLY LUMINOUS EXTRAGALACTIC NOVA SN 2010U
}

\author{
Ian Czekala $^{1}$, E. Berger ${ }^{1}$, R. Chornock $^{1}$, A. Pastorello ${ }^{2}$, G. H. Marion ${ }^{1,3}$, R. Margutti $^{1}$, M. T. Botticella ${ }^{4}$, \\ P. Challis ${ }^{1}$, M. Ergon ${ }^{5}$, S. SmartT $^{6}$, J. Sollerman ${ }^{5}$, J. Vinkó $^{7}$, and J. C. Wheeler ${ }^{3}$ \\ ${ }^{1}$ Harvard-Smithsonian Center for Astrophysics, 60 Garden Street MS 10, Cambridge, MA 02138, USA; iczekala@cfa.harvard.edu \\ 2 INAF-Osservatorio Astronomico di Padova, Vicolo dellOsservatorio 5, I-35122 Padova, Italy \\ ${ }^{3}$ Department of Astronomy, The University of Texas at Austin, RLM 5.208, Austin, TX 78712-1081, USA \\ ${ }^{4}$ INAF-Osservatorio Astronomico di Capodimonte Salita Moiariello 16, I-80131 Napoli, Italy \\ ${ }^{5}$ The Oskar Klein Centre, Department of Astronomy, AlbaNova, Stockholm University, SE-10691 Stockholm, Sweden \\ ${ }^{6}$ Astrophysics Research Centre, School of Mathematics and Physics, Queen's University Belfast, Belfast BT7 1NN, UK \\ ${ }^{7}$ Department of Optics and Quantumelectronics, University of Szeged, Szeged, Hungary \\ Received 2012 October 4; accepted 2013 January 11; published 2013 February 15
}

\begin{abstract}
We present observations of the unusual optical transient SN 2010U, including spectra taken 1.03 days to 15.3 days after maximum light that identify it as a fast and luminous Fe II type nova. Our multi-band light curve traces the fast decline $\left(t_{2}=3.5 \pm 0.3\right.$ days $)$ from maximum light $\left(M_{V}=-10.2 \pm 0.1 \mathrm{mag}\right)$, placing SN $2010 \mathrm{U}$ in the top $0.5 \%$ of the most luminous novae ever observed. We find typical ejecta velocities of $\approx 1100 \mathrm{~km} \mathrm{~s}^{-1}$ and that SN $2010 \mathrm{U}$ shares many spectral and photometric characteristics with two other fast and luminous Fe II type novae, including Nova LMC 1991 and M31N-2007-11d. For the extreme luminosity of this nova, the maximum magnitude versus rate of decline relationship indicates a massive white dwarf (WD) progenitor with a low pre-outburst accretion rate. However, this prediction is in conflict with emerging theories of nova populations, which predict that luminous novae from massive WDs should preferentially exhibit an alternate spectral type $(\mathrm{He} / \mathrm{N})$ near maximum light.
\end{abstract}

Key words: novae, cataclysmic variables - supernovae: individual (SN 2010U) - X-rays: stars

Online-only material: color figures

\section{INTRODUCTION}

Unprecedented areal and temporal coverage of the sky from dedicated surveys and amateur observers has greatly amplified the discovery rate of unusual optical transients. Surveys such as Pan-STARRS, the Palomar Transient Factory, and the Catalina Real-Time Transient Survey have demonstrated the wealth of data that will be common in the era of the Large Synoptic Survey Telescope (LSST). In particular, a previously sparse regime of transient phase space between classical novae $(\mathrm{CNe}$; $M_{V \text {, peak }} \sim-8 \mathrm{mag}$; Bode \& Evans 2008) and supernovae $\left(M_{V \text {, peak }} \sim-18 \mathrm{mag}\right.$; Filippenko 1997) is now being populated with an increasing number of transients. These objects are quite diverse in their properties and may shed light on a wide range of explosion and eruption physics.

In recent years, objects like SN 2008S and NGC 300 OT (Prieto et al. 2008; Botticella et al. 2009; Berger et al. 2009; Thompson et al. 2009; Szczygieł et al. 2012) and other luminous blue variables (LBVs; Humphreys \& Davidson 1994; Pastorello et al. 2010; Smith et al. 2011) have been subjected to intense scrutiny. These intermediate luminosity optical transients (ILOTs; also referred to as SN impostors and luminous red novae) might be the eruption of a dust-enshrouded massive star and promise to lend great insight into the late stages of massive stellar evolution or other poorly understood stellar physics. Because the phase space these eruptions inhabit is crowded with fundamentally different transient systems, it is important for future transient discovery to scrutinize this region with intensive spectroscopic and photometric followup to distinguish these explosions from other more traditional explosions, such as $\mathrm{CNe}$ or supernovae, which to fall in this region of phase space would qualify them as remarkable in their own right.

$\mathrm{CNe}$ are binary systems where there is mass transfer from a (possibly evolved) secondary through the L1 Lagrange point to a degenerate white dwarf (WD). When enough material has accreted to obtain critical temperature and density, nuclear burning begins. The $p-p$ chain gives way to $\mathrm{CNO}$ reactions, which drive convection. The amount of energy deposited by the $\beta^{+}$unstable nuclei then drives a radiative wind. Because degenerate matter on the surface of the WD has an equation of state independent of temperature, these reactions proceed in a runaway fashion until the Fermi temperature is reached and the surface layers of the WD begin to function as an ideal gas sensitive to temperature and finally expand. This expansion speed can easily reach escape velocity and the radiation pressure ejects a shell of material (Warner 2003).

Within our Milky Way, interstellar extinction and selection effects limit the number of observed novae, making determination of the total Galactic nova rate imprecise. Previous studies determined the rate to be as high as $73 \pm 24 \mathrm{yr}^{-1}$ (Liller \& Mayer 1987), although recent investigations have converged upon the rate of $35 \pm 11 \mathrm{yr}^{-1}$ via direct measurement (Shafter 1997) of Milky Way novae and indirect comparison (Darnley et al. 2006) of the Galaxy to M31 (Darnley et al. 2006). The mean absolute magnitude of novae is $M_{V} \approx-7.5 \mathrm{mag}$, and of nearly a thousand novae on record, less than 10 reached peak absolute magnitude brighter than $M_{V}=-10.0$ mag (Shafter et al. 2009).

Here we present the detailed photometric and spectroscopic observations of SN 2010U. We show that SN 2010U is clearly super-Eddington at maximum light and identify it as a close spectroscopic analog to other super-Eddington novae. We compare SN 2010U to the general nova population and recognize it as one of the most luminous and fast declining novae discovered to date. These characteristics of SN 2010U make it a valuable object to study in the context of outburst models and progenitor studies of luminous novae.

\section{DISCOVERY AND REDUCTION}

Nakano \& Kadota (2010) discovered SN 2010U in NGC 4214 on 2010 February 5.63 UT (UT dates are used throughout 


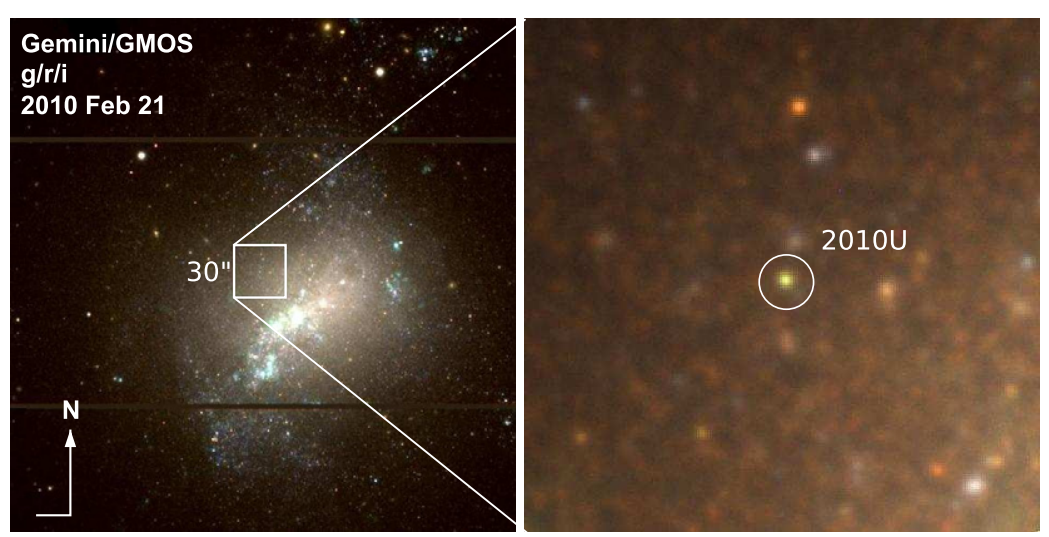

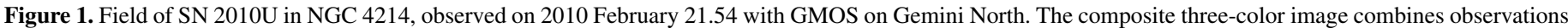
with gri filters. The inset on the right shows SN $2010 \mathrm{U}$ about two weeks after maximum light.

(A color version of this figure is available in the online journal.)

this paper) and was subsequently observed by several amateur astronomers that night. SN $2010 \mathrm{U}$ is located at R.A. = $12^{\mathrm{h}} 15^{\mathrm{m}} 41^{\mathrm{s}} .06$, decl. $=+36^{\circ} 20^{\prime} 02^{\prime \prime} .9(\mathrm{~J} 2000)$, about $20^{\prime \prime}$ east and $27^{\prime \prime}$ north of the center of NGC 4214 (Nakano \& Kadota 2010; Figure 1). We use the distance modulus of $m-M=27.41 \pm$ 0.03 mag (Dalcanton et al. 2009) for NGC 4214 and correct all magnitudes for Galactic reddening of $E(B-V)=0.02 \mathrm{mag}$ using the dust maps of Schlegel et al. (1998). Observations by Itagaki provide a pre-explosion limit of $18.8 \mathrm{mag}$ (unfiltered) on 2010 January 24.74. Humphreys et al. (2010) determined that SN 2010U was initially mis-classified as a supernova, and is in fact a luminous and fast CN. They conclude that SN 2010U reached a peak absolute magnitude of $M_{R}=-10.5 \mathrm{mag}$ and faded two magnitudes on a timescale of $t_{2} \approx 15$ days. They use a distance modulus of $m-M=27.53$, while our distance modulus determination is more recent. Adopting our distance modulus, the peak absolute magnitude using the results of Humphreys et al. (2010) is $M_{R}=-10.4 \mathrm{mag}$.

\subsection{Photometry}

We initiated a multi-band photometric follow-up campaign of SN 2010U starting on 2010 February 6.98 using the $2 \mathrm{~m}$ Liverpool Telescope (LT; Steele et al. 2004) with RatCam; the $8 \mathrm{~m}$ Gemini North Telescope (GN) with Gemini Multi-Object Spectrograph (GMOS; Hook et al. 2004); and the $2.56 \mathrm{~m}$ Nordic Optical Telescope (NOT; Djupvik \& Andersen 2010) with the Andalucia Faint Object Spectrograph and Camera (ALFOSC). We also collected photometry from amateur astronomers $\mathrm{K}$. Itagaki, T. Yusa, J. Brimacombe, and J. Nicolas, who kindly provided to us their unfiltered discovery images from 2010 February 5.65 to 13.03 , which captured the rise and peak of SN 2010U.

We bias-subtracted and flat-fielded all images using standard techniques in IRAF, and determined instrumental magnitudes using point spread function (PSF) fitting of the source. We obtained nightly zero points by observing a number of standard fields from the Landolt (1992) catalog. We improved the calibration of individual magnitudes of the transient through comparison with the average magnitudes of a local stellar sequence in the field of SN 2010U established during selected photometric nights.

Observations from the LT used Landolt $B$ and $V$ band and Sloan $r^{\prime}$ and $i^{\prime}$ band, but were calibrated to Landolt standards in the Vega system. To place all fluxes on the same system, we transformed these measurements to the AB system using offsets derived from pysynphot ${ }^{8}$ of $-0.115,0.000$, +0.142 , and +0.356 mag, respectively. Unfiltered observations with NOT, as well as amateur observations, were unfiltered but initially calibrated to Vega $R$ band, so we transformed these measurements to $\mathrm{AB}$ using an offset of $+0.183 \mathrm{mag}$. Since GN observations were initially calibrated to the AB system no transformation was necessary, and all magnitudes quoted in this paper are $\mathrm{AB}$ unless otherwise noted. The quantum efficiency curves of the instrumental configurations used by the amateur astronomers peak around 6000-6200 $\AA$, so the unfiltered magnitudes were scaled to match the $r^{\prime}$-band photometry. In the case of the observation by J. Brimacombe, however, the transmission curve of his luminance filter ${ }^{9}$ peaks at $5500 \AA$ and sharply declines outside the range 4200-6800 , making calibration to $V$ band most appropriate. We summarize the optical photometric measurements in Table 1 and present the light curve in Figure 2.

We also observed SN 2010U with the Swift satellite (Gehrels et al. 2004) on 2010 March 3.82 with the X-Ray Telescope (XRT; Burrows et al. 2005) and the UV/Optical Telescope (UVOT; Roming et al. 2005). We did not detect any X-ray or UV/optical emission coincident with the location of the source. A previous Swift/XRT observation of NGC 4214 on 2007 March 26.50, which included the field of SN 2010U, showed no activity coincident with the source location. We analyzed all Swift data with the Heasoft -6.11 software package and corresponding calibration files, applying standard screening and filtering criteria. We reduced XRT data with the xrtpipeline and determined $3 \sigma$ upper limits with the sosta task in the ximage suite using a $5^{\prime \prime}$ radius aperture (see Table 2). We processed UVOT with the standard UVOT data reduction pipeline (Poole et al. 2008) and determined $3 \sigma$ upper limits with a 5 " radius aperture (see Table 3 ).

\subsection{Spectroscopy}

We obtained three low-resolution optical spectra of SN 2010U using the Marcario Low-Resolution Spectrograph (LRS; Hill et al. 1998) on the Hobby-Eberly Telescope (HET; Ramsey et al. 1998), ALFOSC on NOT, and GMOS on GN. We reduced the NOT spectrum using the QUBA pipeline (Valenti et al. 2011), implemented in IRAF, and the HET and GMOS spectra using

\footnotetext{
8 http://stsdas.stsci.edu/pysynphot/

9 Transmission function at http://www.sbig.com/site/sbwhtmls/ announcement_baader_narrowband_f $2 . h t m$
} 
Table 1

Photometry of SN 2010 U

\begin{tabular}{|c|c|c|c|c|c|}
\hline 2010 UT & Filter & $\begin{array}{c}m \pm \sigma_{m} \\
\text { (Vega) }\end{array}$ & $\begin{array}{c}m \pm \sigma_{m} \\
(\mathrm{AB}, \text { de-ext })\end{array}$ & $\begin{array}{c}f_{v} \\
(\mu \mathrm{Jy} \pm \sigma)\end{array}$ & Observer/Telescope \\
\hline Jan 24.74 & $R$ & $>18.80$ & $>18.92$ & $<98$ & Itagaki unfiltered \\
\hline Feb 5.65 & $R$ & $17.38 \pm 0.15$ & $17.50 \pm 0.15$ & $362 \pm 86$ & Itagaki unfiltered \\
\hline Feb 6.27 & $V$ & $17.28 \pm 0.09$ & $17.20 \pm 0.09$ & $477 \pm 66$ & Brimacombe unfiltered \\
\hline Feb 6.34 & $R$ & $17.10 \pm 0.20$ & $17.23 \pm 0.21$ & $466 \pm 153$ & Yusa unfiltered \\
\hline Feb 6.57 & $R$ & $17.21 \pm 0.27$ & $17.33 \pm 0.27$ & $424 \pm 180$ & Itagaki unfiltered \\
\hline Feb 6.98 & $R$ & $17.52 \pm 0.16$ & $17.65 \pm 0.16$ & $317 \pm 82$ & Nicolas \\
\hline Feb 6.98 & $B$ & $18.09 \pm 0.04$ & $17.88 \pm 0.04$ & $255 \pm 17$ & LT \\
\hline Feb 6.98 & $V$ & $17.64 \pm 0.05$ & $17.57 \pm 0.05$ & $341 \pm 27$ & $\mathrm{LT}$ \\
\hline Feb 6.98 & $r^{\prime}$ & $17.54 \pm 0.04$ & $17.62 \pm 0.04$ & $325 \pm 20$ & LT \\
\hline Feb 6.98 & $i^{\prime}$ & $17.42 \pm 0.04$ & $17.73 \pm 0.04$ & $294 \pm 20$ & LT \\
\hline Feb 8.98 & $B$ & $18.90 \pm 0.12$ & $18.69 \pm 0.12$ & $121 \pm 23$ & LT \\
\hline Feb 8.98 & V & $18.82 \pm 0.08$ & $18.74 \pm 0.08$ & $115 \pm 15$ & LT \\
\hline Feb 8.98 & $r^{\prime}$ & $18.48 \pm 0.08$ & $18.56 \pm 0.08$ & $136 \pm 18$ & LT \\
\hline Feb 8.98 & $i^{\prime}$ & $18.42 \pm 0.07$ & $18.73 \pm 0.07$ & $117 \pm 13$ & $\mathrm{LT}$ \\
\hline Feb 10.07 & $B$ & $19.59 \pm 0.08$ & $19.38 \pm 0.08$ & $64 \pm 8$ & $\mathrm{LT}$ \\
\hline Feb 10.07 & $V$ & $19.43 \pm 0.07$ & $19.36 \pm 0.07$ & $65 \pm 7$ & LT \\
\hline Feb 10.07 & $r^{\prime}$ & $19.01 \pm 0.04$ & $19.09 \pm 0.04$ & $84 \pm 5$ & LT \\
\hline Feb 10.07 & $i^{\prime}$ & $18.96 \pm 0.06$ & $19.27 \pm 0.06$ & $71 \pm 7$ & LT \\
\hline Feb 10.17 & $i^{\prime}$ & $18.99 \pm 0.04$ & $19.30 \pm 0.04$ & $69 \pm 4$ & LT \\
\hline Feb 10.19 & $R$ & $19.04 \pm 0.14$ & $19.16 \pm 0.14$ & $78 \pm 17$ & NOT unfiltered \\
\hline Feb 11.10 & $r^{\prime}$ & $19.32 \pm 0.07$ & $19.40 \pm 0.07$ & $63 \pm 7$ & LT \\
\hline Feb 12.20 & $R$ & $19.44 \pm 0.10$ & $19.56 \pm 0.10$ & $54 \pm 9$ & NOT unfiltered \\
\hline Feb 13.03 & $R$ & $19.55 \pm 0.40$ & $19.67 \pm 0.40$ & $49 \pm 31$ & Nicolas \\
\hline Feb 21.54 & $g^{\prime}$ & $\ldots$ & $21.15 \pm 0.05$ & $13 \pm 1$ & GMOS \\
\hline Feb 21.54 & $r^{\prime}$ & $\ldots$ & $20.18 \pm 0.11$ & $31 \pm 5$ & GMOS \\
\hline Feb 21.54 & $i^{\prime}$ & $\ldots$ & $20.35 \pm 0.08$ & $26 \pm 3$ & GMOS \\
\hline Feb 23.98 & $B$ & $>20.89$ & $>20.68$ & $<19$ & LT \\
\hline Feb 23.98 & $V$ & $20.89 \pm 0.24$ & $20.82 \pm 0.24$ & $17 \pm 7$ & LT \\
\hline Feb 23.98 & $r^{\prime}$ & $20.17 \pm 0.14$ & $20.26 \pm 0.14$ & $29 \pm 6$ & LT \\
\hline Feb 23.98 & $i^{\prime}$ & $20.32 \pm 0.18$ & $20.63 \pm 0.18$ & $20 \pm 6$ & LT \\
\hline
\end{tabular}

Notes. Unfiltered images were calibrated to Landolt $R$ band using field stars from the Landolt catalog. One exception to this was the unfiltered image from J. Brimacombe on 2010 February 6.27 UT, which was calibrated to standard $V$ band (see Section 2.1). Raw photometry is reported in the Vega system, uncorrected for Galactic or host galaxy extinction. To put all measurements on a uniform scale, the Vega measurements were converted to $\mathrm{AB}$ magnitudes using the formula $m_{\mathrm{x}, \mathrm{AB}}=m_{x, \mathrm{VEGA}}+\Delta m_{\mathrm{x}}$, where $\Delta m_{\mathrm{x}}$ is the offset derived from pysynphot: $\Delta m_{B}=-0.115, \Delta m_{V}=0.000, \Delta m_{R}=0.183, \Delta m_{r^{\prime}}=0.142$, and $\Delta m_{i^{\prime}}=0.356$. These AB magnitude and Janksy values in the table are corrected for galactic reddening of $E(B-V)=0.022$, while the Vega values are uncorrected. Observations that were originally in $\mathrm{AB}$ were not converted back to Vega.

Table 2

Swift XRT Observations of SN 2010U

\begin{tabular}{lcccc}
\hline \hline UT Date & $\begin{array}{c}\text { Exposure } \\
(\mathrm{s})\end{array}$ & $\begin{array}{c}\text { Counts } \\
\left(\text { counts s}^{-1}\right)\end{array}$ & $\begin{array}{c}\text { Flux } \\
\left(\mathrm{erg} \mathrm{s}^{-1}\right)(k T=60 \mathrm{eV})\end{array}$ & $\begin{array}{c}\text { Flux } \\
\left(\mathrm{erg} \mathrm{s}^{-1}\right)(k T=5 \mathrm{keV})\end{array}$ \\
\hline 2007 Mar 26.50 UT & 5624 & $<6.67 \mathrm{E}-03$ & $<1.9 \times 10^{-13}$ & $<1.1 \times 10^{-12}$ \\
2010 Mar 3.82 UT & 1903 & $<9.08 \mathrm{E}-03$ & $<2.6 \times 10^{-13}$ & $<1.5 \times 10^{-12}$ \\
\hline
\end{tabular}

Notes. Energy band $0.3-10.0 \mathrm{keV}$. All upper limits are $3 \sigma$.

Table 3

Swift UVOT Photometry of SN 2010 U

\begin{tabular}{lcccc}
\hline \hline $\begin{array}{l}\text { UT Date } \\
(2010 \text { March })\end{array}$ & Filter & $\begin{array}{c}\lambda \\
(\AA)\end{array}$ & $\begin{array}{c}\text { Exposure } \\
(\mathrm{s})\end{array}$ & $\begin{array}{c}m \\
(\mathrm{AB})\end{array}$ \\
\hline 3.97 & UVM2 & 2246 & 465 & $>20.63$ \\
3.82 & UVW1 & 2600 & 594 & $>20.88$ \\
3.82 & $U$ & 3465 & 141 & $>19.82$ \\
3.82 & $B$ & 4392 & 141 & $>19.15$ \\
3.97 & $V$ & 5468 & 60 & $>17.88$ \\
\hline
\end{tabular}

Notes. All observations taken 2010 March 3.82 UT. All upper limits are $3 \sigma$. standard tasks in IRAF. We observed all targets at low airmass $(\lesssim 1.2)$ with the slit was aligned to the parallactic angle, and fluxcalibrated each spectrum using a spectrophotometric standard star observed at a similar airmass. All spectra were wavelengthcalibrated by comparison with helium-neon-argon arc lamps. We summarize spectroscopic measurements and instrumental configurations in Table 4 and identify spectral lines in Table 5. We analyzed the resulting one-dimensional spectroscopic data in IRAF using onedspec tasks and the Scipy Python packages (Jones et al. 2001). 
Table 4

SN 2010U Spectroscopic Observations

\begin{tabular}{llcccccc}
\hline \hline $\begin{array}{l}\Delta t^{\mathrm{a}} \\
(\text { days })\end{array}$ & 2010 UT & Telescope & Instrument & $\begin{array}{c}\lambda \\
(\AA)\end{array}$ & $\begin{array}{c}\text { Resolution } \\
(\AA)\end{array}$ & $\begin{array}{c}\text { Exposure } \\
(\mathrm{A})\end{array}$ & $\begin{array}{c}\text { Airmass } \\
\text { Slit Width } \\
(\operatorname{arcsec})\end{array}$ \\
\hline+1.03 & Feb 7.30 & HET & LRS & $4200-10000$ & 15 & 437 & 1.23 \\
+5.94 & Feb 12.21 & NOT & ALFOSC & $3200-9100$ & 15 & $3 \times 900$ & 1.05 \\
+15.30 & Feb 21.57 & GN & GMOS & $4700-8840$ & 8 & $2 \times 1200$ & 1.07 \\
\hline
\end{tabular}

Notes. Maximum light was on 2010 February 6.27.

${ }^{\mathrm{a}} \Delta t=t-t_{\max }$, where $t_{\max }=2010$ February 6.27 UT.

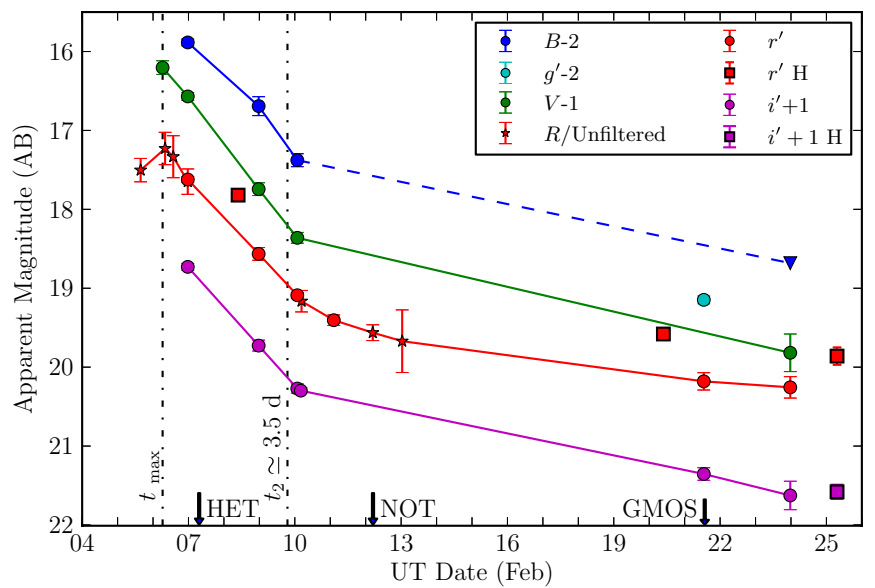

Figure 2. Light curves of SN $2010 \mathrm{U}$ corrected for Galactic extinction (Schlegel et al. 1998) and plotted in AB magnitudes. $B$-, $V$-, and $i^{\prime}$-band data points are offset for clarity, and unfiltered amateur observations are calibrated to $R$ band. We adopt the date of $V$-band maximum, 2010 February 6.27, as the date of maximum light for SN 2010U at apparent magnitude of $17.28 \mathrm{mag}$. The $g^{\prime}$-band measurement is distinct from nearby $B$ - and $V$-band measurements because the differences in the passbands yield significant deviations due to the presence of emission lines (Figure 6). Photometry from Humphreys et al. (2010) is converted to the $\mathrm{AB}$ system, corrected for extinction, and included in this light curve. We find a $\sim 0.3$ mag difference in $r^{\prime}$ band, which is the source of disagreement in our determination of $t_{2}$. However, we note that the very rapid decline is also apparent in $B, V$, and $i^{\prime}$ band.

(A color version of this figure is available in the online journal.)

\section{RESULTS}

Complete photometric and spectroscopic coverage of SN 2010 U confirms the findings of Humphreys et al. (2010): SN 2010U is a luminous CN, exhibiting a rapid optical decline and evolution from an optically thick spectrum dominated by hydrogen and iron emission lines to an optically thin nova spectrum entering the nebular stage. SN 2010U is not a supernova nor the eruption of a massive star because of its modest ejecta velocities $\left(\approx 1100 \mathrm{~km} \mathrm{~s}^{-1}\right)$ and rapid optical decline and spectral evolution. Supernovae typically exhibit much higher expansion velocities ( $\gtrsim 10^{4} \mathrm{~km} \mathrm{~s}^{-1}$; Filippenko 1997) and while LBV eruptions exhibit a range of expansion velocities $\left(\sim 200-2000 \mathrm{~km} \mathrm{~s}^{-1}\right.$; Smith et al. 2011) and strong hydrogen Balmer emission, the presence of CNO element lines and rapid optical and spectral evolution of SN 2010U strongly indicate that it was a CN and not an LBV.

\subsection{Optical Photometric Evolution}

SN 2010U evolved rapidly after its discovery on 2010 February 5.63. Our light curve is well sampled near maximum light in $r^{\prime}$ band, and the transient is seen to rise $\approx 0.25 \mathrm{mag}$ from discovery to maximum. Although the rise of SN 2010U is not captured in $V$ band, the first measurement at $17.28 \mathrm{mag}$ is contemporaneous with the measurement of maximum light in $r^{\prime}$ band. For the purpose of comparison with previous events, we adopt the date of $V$-band maximum, 2010 February 6.27, as the date of maximum light. After maximum, SN 2010U rapidly declined with a linear slope in magnitude space, at first steeply and then becoming more gradual after 2010 February 10 (Figure 2). We followed the light curve of the transient until 2010 February 24. Humphreys et al. (2010) followed the transient until 2010 March 18.40, reporting a continued steady decline.

The photometric evolution of $\mathrm{CNe}$ is typically parameterized by the time to decline by two magnitudes from maximum light, $t_{2}$. Several studies have shown that $B$ or $V$ band are most appropriate to measure $t_{2}$ because $\mathrm{H} \alpha$ emission complicates measurements in $r^{\prime}$ band (Shafter et al. 2011; Bode \& Evans 2008). We measure $t_{2}$ by adopting the $V$-band maximum (2010 February 6.27), and then linearly interpolating between two $V$-band measurements at 2010 February 8.98 and 2010 February 10.07 in magnitude space, which gives a result that is accurate to \pm 0.13 days. The uncertainty in the date of maximum light derives from the assumption that maximum light in $V$ band corresponds with maximum light in $r^{\prime}$ band, and therefore we have captured the peak of the light curve to \pm 0.3 days. We find that SN 2010U underwent a fast decline, with a $V$-band maximum of $M_{V}=-10.2 \pm 0.1 \mathrm{mag}$ and $t_{2}=3.5 \pm 0.3$ days. Our determination of absolute magnitude and $t_{2}$ are in contrast to the results of Humphreys et al. (2010), who derive $M_{R, \max } \approx$ $-10.5 \mathrm{mag}$ and $t_{2} \approx 15$ days. This is primarily due to the smaller distance modulus adopted here, and our better sampling of the light curve in the range $t_{\max }$ to $t_{\max }+15$ days. In addition, a fast decline is evident in $B, V$, and $i^{\prime}$ band as well. The rise time of SN 2010U from quiescence to maximum light remains unconstrained due to the comparatively shallow upper limit $\left(m_{r^{\prime}} \approx 18.8 \mathrm{mag}\right.$ ) on January $24.74 \mathrm{UT}$ and a large gap before discovery.

We compare the colors of SN 2010U to those of other fast and luminous novae and the general nova population to determine whether there is intrinsic host galaxy extinction. In Figure 3, the colors of SN $2010 \mathrm{U}$ are plotted against another fast and luminous nova, Nova LMC 1991 (hereafter L91), and the average colors of the nova population. van den Bergh \& Younger (1987) find that of seven novae at maximum light, $(B-V)_{\mathrm{avg}}^{\max }=0.23 \pm 0.06 \mathrm{mag}$, with a dispersion $\sigma_{B-V} \lesssim$ $0.16 \mathrm{mag}$. They also find that at $t_{2}, 13$ novae are found to have an intrinsic color $(B-V)_{\mathrm{avg}}^{t_{2}}=-0.02 \pm 0.04 \mathrm{mag}$, with a dispersion $\sigma_{B-V} \lesssim 0.12 \mathrm{mag}$.

It is interesting to compare SN $2010 \mathrm{U}$ to L91 and speculate that any color difference might be due to intrinsic host galaxy extinction. For SN 2010U near maximum light $(B-V)=$ $0.43 \pm 0.06 \mathrm{mag}$ and near $t_{2}(B-V)=0.13 \pm 0.11 \mathrm{mag}$. If we were to assume that SN 2010U has the same intrinsic colors as L91, then SN 2010U might suffer as much as $E(B-V) \approx 0.2 \mathrm{mag}$ of additional intrinsic extinction, which 


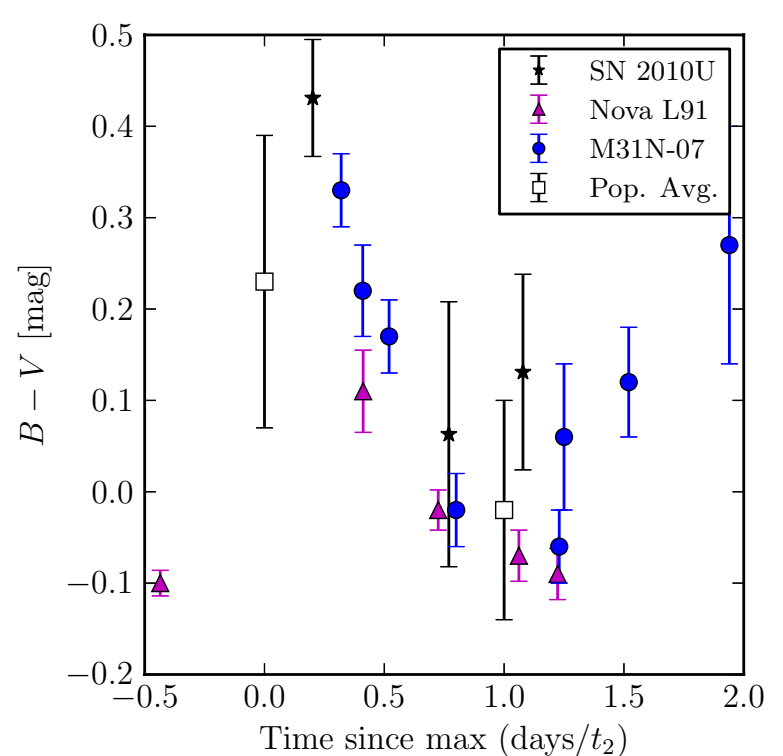

Figure 3. Colors of SN 2010U, L91, M31N, and the general nova population (van den Bergh \& Younger 1987) in units of $t_{2}$ after correction for Galactic reddening. A comparison of SN 2010U to L91, another luminous and fast nova, assuming that any color difference is due to intrinsic host galaxy extinction, indicates that SN $2010 \mathrm{U}$ might suffer as much as $E(B-V) \approx 0.2 \mathrm{mag}$ of additional intrinsic extinction, raising the peak luminosity to $M_{V} \approx-10.9 \mathrm{mag}$. For the purpose of comparison, all colors are given in Vega magnitudes.

(A color version of this figure is available in the online journal.)

would raise its peak brightness to $M_{V} \approx-10.9$ mag, making it the most luminous CN on record. However, SN $2010 \mathrm{U}$ could also simply be intrinsically redder than L91.

Humphreys et al. (2010) assume the $V-R \approx 1.1$ mag color of V1500 Cyg, another luminous nova, to infer $M_{V} \approx-9.4 \mathrm{mag}$ for SN 2010U. However, we measure $V-r^{\prime}=0.1 \mathrm{mag}$ (Vega), suggesting that SN $2010 \mathrm{U}$ was not as red as V1500 Cyg. We conservatively assume no intrinsic host galaxy extinction for all further analysis.

\subsection{X-Ray}

The Swift XRT and UVOT observed the location of SN 2010U 25.6 days after maximum optical light and did not detect the source (Tables 2 and 3), placing a $3 \sigma$ upper limit of $9.1 \times 10^{-3}$ counts s $^{-1}$ for the X-rays in the $0.3-10.0 \mathrm{keV}$ energy band. Using the relationship derived in Güver \& Özel (2009) to convert optical extinction $A_{V}$ into hydrogen column density $N_{\mathrm{H}}$, we obtain $N_{\mathrm{H}}\left(\mathrm{cm}^{-2}\right)=(2.21 \pm 0.09) \times 10^{21} A_{V}(\mathrm{mag})=$ $1.60 \times 10^{20} \mathrm{~cm}^{-2}$. Using the Chandra X-ray Center's Portable, Interactive Multi-Mission Simulator (PIMMS), ${ }^{10}$ and assuming a spectrum for the nova X-ray emission, we convert count rates into flux limits by assuming a spectrum for the nova.

Schwarz et al. (2011) present a compilation of 52 Galactic and Magellanic Cloud CNe and recurrent novae (RNe) observed with the Swift XRT, and Henze et al. $(2010,2011)$ present a compilation of M31 novae observed with XMM-Newton and Chandra. X-ray studies of $\mathrm{CNe}$ have identified two different emission components, a hard X-ray component and a soft $\mathrm{X}$-ray component. The fastest optically declining novae (as measured by $t_{2}$ ) usually have an early hard X-ray phase, while the slower novae do not. The hard X-ray emission may originate from shocks between the fast moving ejecta and preexisting circumstellar material, and typically is hard thermal

\footnotetext{
10 http://cxc.harvard.edu/toolkit/pimms.jsp
}

bremsstrahlung ( $\left.T \sim 6 \times 10^{7}-1 \times 10^{8} \mathrm{~K}\right)$, low luminosity $\left(\sim 10^{34} \mathrm{erg} \mathrm{s}^{-1}\right.$; Balman et al. 1998), and of shorter duration than the soft X-ray phase (Schwarz et al. 2011). The soft phase begins when the nova shell becomes optically thin and the photosphere of the nova recedes to the surface of the hot WD, with blackbody emission at $T=2-8 \times 10^{5} \mathrm{~K}$ (Schwarz et al. 2011), called Super Soft Source (SSS) "turn-on" (Henze et al. 2010). This emission lasts as long as nuclear reactions continue on the surface of the WD. Schwarz et al. (2011) and Henze et al. (2011) find that the Super Soft X-ray phase begins and ends ("SSS turn-off") sooner for fast novae (as measured by $t_{2}$ ) than for slow novae and that novae with slower expansion velocities will enter the Super Soft state later but emit X-rays for longer. The correlation between Super Soft X-ray turn off time and $t_{2}$ has significant scatter (Hachisu \& Kato 2010; Schwarz et al. 2011), but if for SN 2010U $t_{2}=3.5 \pm 0.3$ days, then the turn-off time would be lower than 60 days and possibly as low as 10 days.

Adopting a temperature of $k T=5 \mathrm{keV}$ for the hard component of SN 2010U would place a $3 \sigma$ upper limit on the $\mathrm{X}$-ray luminosity of $L_{X}=1.6 \times 10^{39} \mathrm{erg} \mathrm{s}^{-1}$, while adopting a temperature of $k T=60 \mathrm{eV}$ for the soft component of SN 2010U would place a limit of $L_{X}=2.6 \times 10^{38} \mathrm{erg} \mathrm{s}^{-1}$. While neither of these limits are strong constraints, the upper limit on the Super Soft emission approaches the X-ray luminosities of some novae on record. Schwarz et al. (2011) found that for Nova V407 Cyg the blackbody luminosity of the Super Soft emission was $L_{X}=9.3 \times 10^{37} \mathrm{erg} \mathrm{s}^{-1}$ at 27 days after optical maximum, and that nuclear burning on the surface of the WD occurred from eruption until about 30 days after optical maximum, meaning that SN 2010U could not have been much brighter in X-rays than Nova V407 Cyg. The deeper pre-explosion observation on 2007 March 26.50 of the field of SN 2010U placed a $3 \sigma$ upper limit of $6.7 \times 10^{-3}$ counts $\mathrm{s}^{-1}$, providing a weak upper limit on the luminosity of the nova system in quiescence. Adopting a temperature of $k T=60 \mathrm{eV}$ for a soft quiescent spectrum of SN 2010U would place a limit of $L_{X}=2.1 \times 10^{38} \mathrm{erg} \mathrm{s}^{-1}$.

\subsection{Bolometric Flux Evolution}

During the early evolution of the light curve near maximum light ( $t \lesssim 4$ days), the ejected shell of SN 2010U is still optically thick and we can fit the spectral energy distribution (SED; Figure 4) with a spherical blackbody function. We use the photometry from 2010 February 6.98, 8.98, and 10.07. Effective wavelengths for these filters were determined using pysynphot and the HET and NOT spectra, yielding $\lambda_{B \text {,eff }}=4387 \AA$, $\lambda_{V \text {, eff }}=5468 \AA, \lambda_{r^{\prime}, \text { eff }}=6202 \AA$, and $\lambda_{i^{\prime}, \text { eff }}=7463 \AA$.

We use $\chi^{2}$ minimization to find the best-fit parameters of radius and temperature, shown in Figure 4. For the SED nearest maximum light (2010 February 6.98), we obtain a photospheric temperature of $T=8090 \pm 470 \mathrm{~K}$ and a radius of $R=1.99 \pm 0.19$ AU. Within the errors, the temperature of the photosphere remains constant for the following two epochs, while its radius recedes to $\approx 1.0 \mathrm{AU}$, indicating that the envelope becomes optically thin. This temperature fits well with the typical $T_{\text {eff }} \leqslant 10^{4} \mathrm{~K}$ derived for novae at visual maximum (Williams 1992).

If we combine the expansion velocity measured from spectral lines (see Section 3.4) with the radius of the photosphere and assume ballistic expansion, we can estimate the time since explosion. We determine that time from explosion to 2010 February 6.98 was $t=3.06 \pm 0.40$ days. This suggests a rapid rise to maximum, but is otherwise consistent with the observations since it is uncertain how accurately the spectral 
Table 5

SN 2010U Line Identifications

\begin{tabular}{|c|c|c|c|c|c|}
\hline Line & Date & $\begin{array}{l}\text { Wavelength } \\
(\AA)\end{array}$ & $\begin{array}{l}\text { EW } \\
(\AA)\end{array}$ & $\begin{array}{c}\text { FWHM } \\
(\AA)\end{array}$ & $\begin{array}{l}\text { Velocity } \\
\left(\mathrm{kms}^{-1}\right)^{\mathrm{a}}\end{array}$ \\
\hline \multirow[t]{3}{*}{$\mathrm{H} \alpha \lambda 6562.85$} & Feb 7.30 & $6565.0 \pm 1.6$ & $-74.7 \pm 4.8$ & $54.1 \pm 3.4$ & $2470 \pm 155$ \\
\hline & Feb 12.21 & $6563.2 \pm 0.6$ & $-812.1 \pm 23.0$ & $46.0 \pm 1.0$ & $2100 \pm 45$ \\
\hline & Feb 21.51 & $6564.7 \pm 0.0$ & $-1225.0 \pm 3.2$ & $34.4 \pm 0.1$ & $1570 \pm 5$ \\
\hline \multirow[t]{3}{*}{$\mathrm{H} \beta \lambda 4860.36$} & Feb 7.30 & $4867.5 \pm 1.3$ & $-36.1 \pm 2.8$ & $34.3 \pm 2.6$ & $2115 \pm 160$ \\
\hline & Feb 12.21 & $4861.6 \pm 0.7$ & $-296.4 \pm 14.0$ & $31.2 \pm 1.4$ & $1925 \pm 85$ \\
\hline & Feb 21.51 & $4862.2 \pm 0.1$ & $-201.5 \pm 1.3$ & $20.6 \pm 0.1$ & $1275 \pm 5$ \\
\hline \multirow[t]{2}{*}{$\mathrm{H} \gamma \lambda 4343.49$} & Feb 7.30 & $4347.7 \pm 1.6$ & $-19.8 \pm 2.0$ & $30.1 \pm 3.6$ & $2080 \pm 250$ \\
\hline & Feb 12.21 & $4341.8 \pm 1.3$ & $-81.0 \pm 7.1$ & $28.6 \pm 2.6$ & $1975 \pm 175$ \\
\hline $\mathrm{H} \delta \lambda 4101.77$ & Feb 12.21 & $4103.2 \pm 1.7$ & $-50.2 \pm 5.8$ & $30.1 \pm 4.3$ & $2200 \pm 315$ \\
\hline $\mathrm{H} \epsilon \lambda 3970$ & Feb 12.21 & $3973.6 \pm 1.4$ & $-42.1 \pm 5.2$ & $22.7 \pm 3.2$ & $1715 \pm 240$ \\
\hline $\mathrm{H} \zeta \lambda 3889$ & Feb 12.21 & $3891.1 \pm 1.8$ & $-19.1 \pm 3.9$ & $17.8 \pm 6.4$ & $1370 \pm 495$ \\
\hline Paschen $\iota \lambda 8750.47$ & Feb 7.30 & $8753.8 \pm 4.6$ & $-12.6 \pm 3.8$ & $39.5 \pm 22.9$ & $1350 \pm 785$ \\
\hline $\mathrm{C}_{\mathrm{I}} \lambda 9111$ & Feb 7.30 & $9112.4 \pm 4.6$ & $-28.6 \pm 5.7$ & $50.8 \pm 14.1$ & $1675 \pm 465$ \\
\hline С I $\lambda 9408$ & Feb 7.30 & $9408.0 \pm 3.2$ & $-14.2 \pm 4.3$ & $25.0 \pm 12.2$ & $795 \pm 390$ \\
\hline C I $\lambda 9660$ & Feb 7.30 & $9669.3 \pm 11.4$ & $-12.0 \pm 6.2$ & $38.7 \pm 30.4$ & $1200 \pm 940$ \\
\hline \multirow[t]{4}{*}{ N I/Fe II $\lambda 7452$} & Feb 7.30 & $7414.4 \pm 8.1$ & $8.9 \pm 3.0$ & $55.5 \pm 36.1$ & $-1515 \pm 325^{b}$ \\
\hline & Feb 7.30 & $7476.1 \pm 7.1$ & $-7.5 \pm 3.0$ & $40.6 \pm 30.9$ & $970 \pm 285^{\mathrm{c}}$ \\
\hline & Feb 12.21 & $7465.8 \pm 7.9$ & $-25.4 \pm 8.3$ & $48.2 \pm 24.7$ & $1940 \pm 990$ \\
\hline & Feb 21.51 & $7473.8 \pm 2.2$ & $-46.7 \pm 2.0$ & $109.4 \pm 6.0$ & $4400 \pm 240$ \\
\hline \multirow[t]{4}{*}{$\mathrm{N}_{\mathrm{I}} \lambda 8212$} & Feb 7.30 & $8168.7 \pm 6.0$ & $19.1 \pm 4.1$ & $59.4 \pm 18.0$ & $-1580 \pm 220^{b}$ \\
\hline & Feb 7.30 & $8248.3 \pm 6.0$ & $-12.2 \pm 4.0$ & $33.9 \pm 14.6$ & $1325 \pm 220^{\mathrm{c}}$ \\
\hline & Feb 12.21 & $8231.0 \pm 6.8$ & $-53.1 \pm 10.0$ & $78.8 \pm 24.8$ & $2875 \pm 905$ \\
\hline & Feb 21.51 & $8215.7 \pm 0.6$ & $-110.8 \pm 2.0$ & $72.8 \pm 1.4$ & $2660 \pm 50$ \\
\hline $\mathrm{N}_{\mathrm{I}} \lambda 8692 / \mathrm{Ca}$ II & Feb 7.30 & $8648.7 \pm 9.6$ & $13.4 \pm 4.4$ & $51.8 \pm 26.1$ & $1785 \pm 900$ \\
\hline \multirow[t]{2}{*}{$\mathrm{N}_{\mathrm{I}} \lambda 8692$} & Feb 7.30 & $8720.2 \pm 4.3$ & $-10.3 \pm 3.2$ & $34.1 \pm 23.6$ & $1175 \pm 815$ \\
\hline & Feb 12.21 & $8707.3 \pm 5.4$ & $-63.0 \pm 10.0$ & $67.5 \pm 16.8$ & $2330 \pm 580$ \\
\hline N I 8692/Ca II blend & Feb 21.51 & $8656.6 \pm 0.8$ & $-188.6 \pm 2.4$ & $\ldots$ & $\ldots$ \\
\hline$\left[\mathrm{O}_{\mathrm{I}}\right] \lambda 5577.34$ & Feb 21.51 & $5577.4 \pm 0.4$ & $-21.2 \pm 0.8$ & $21.8 \pm 1.0$ & $1170 \pm 50$ \\
\hline$\left[\mathrm{O}_{\mathrm{I}}\right] \lambda 6300.30$ & Feb 21.51 & $6300.6 \pm 0.6$ & $-31.8 \pm 1.1$ & $35.0 \pm 1.3$ & $1665 \pm 60$ \\
\hline$[\mathrm{OI}] \lambda 6363.78$ & Feb 21.51 & $6367.3 \pm 1.2$ & $-14.9 \pm 1.0$ & $34.4 \pm 3.0$ & $1620 \pm 140$ \\
\hline \multirow[t]{4}{*}{ O I $\lambda \lambda 7773.75$} & Feb 7.30 & $7744.2 \pm 3.2$ & $7.2 \pm 2.3$ & $19.1 \pm 9.1$ & $-1140 \pm 125^{b}$ \\
\hline & Feb 7.30 & $7784.3 \pm 4.2$ & $-17.8 \pm 3.9$ & $34.4 \pm 11.7$ & $405 \pm 160^{\mathrm{c}}$ \\
\hline & Feb 12.21 & $7780.8 \pm 1.9$ & $-220.3 \pm 16.0$ & $53.0 \pm 3.5$ & $2045 \pm 135$ \\
\hline & Feb 21.51 & $7778.5 \pm 0.2$ & $-207.6 \pm 2.1$ & $36.5 \pm 0.3$ & $1410 \pm 15$ \\
\hline \multirow[t]{4}{*}{ O г $\lambda \lambda 8446.46$} & Feb 7.30 & $8410.0 \pm 9.8$ & $5.6 \pm 3.2$ & $\ldots$ & $\ldots$ \\
\hline & Feb 7.30 & $8492.9 \pm 5.2$ & $-5.8 \pm 3.0$ & $29.5 \pm 25.3$ & $\ldots$ \\
\hline & Feb 12.21 & $8449.9 \pm 2.1$ & $-216.9 \pm 15.0$ & $59.7 \pm 4.6$ & $2120 \pm 160$ \\
\hline & Feb 21.51 & $8447.5 \pm 0.1$ & $-668.6 \pm 3.2$ & $43.5 \pm 0.2$ & $1545 \pm 5$ \\
\hline O I $\lambda 9264$ & Feb 7.30 & $9268.1 \pm 3.6$ & $-22.8 \pm 5.3$ & $30.5 \pm 9.1$ & $985 \pm 295$ \\
\hline \multirow[t]{2}{*}{$\mathrm{Na}$ I D $\lambda \lambda 5891.94$} & Feb 7.30 & $5868.7 \pm 4.6$ & $8.0 \pm 2.6$ & $21.0 \pm 13.1$ & $-1180 \pm 235^{b}$ \\
\hline & Feb 7.30 & $5906.5 \pm 6.3$ & $-4.5 \pm 2.2$ & $23.4 \pm 17.4$ & $740 \pm 320^{\mathrm{c}}$ \\
\hline \multirow[t]{2}{*}{$\mathrm{Na}$ I D $\lambda 6160$} & Feb 7.30 & $6131.1 \pm 11.5$ & $2.4 \pm 2.2$ & $24.1 \pm 20.8$ & $-1405 \pm 560^{b}$ \\
\hline & Feb 7.30 & $6167.6 \pm 4.1$ & $-7.1 \pm 2.4$ & $21.9 \pm 11.6$ & $370 \pm 200^{c}$ \\
\hline Ca II $\lambda 8498.02$ & Feb 7.30 & $8492.9 \pm 5.2$ & $-5.8 \pm 3.0$ & $29.5 \pm 25.3$ & $1040 \pm 895$ \\
\hline \multirow[t]{2}{*}{ Ca II $\lambda 8542.09$} & Feb 7.30 & $8540.9 \pm 6.9$ & $-6.0 \pm 3.3$ & $\ldots$ & $\ldots$ \\
\hline & Feb 12.21 & $8557.0 \pm 3.9$ & $-28.6 \pm 7.3$ & $35.0 \pm 17.8$ & $1230 \pm 625$ \\
\hline
\end{tabular}

Notes. Maximum light was on 2010 February 6.27 UT. All wavelengths reported are after de-redshifting all spectra using $z=0.00087$. Blue and red velocity-shifted P Cygni components are measured from the rest wavelength of the line species. Measured for where a nice fit was obtained, for low signal to line profiles, only equivalent width is reported. The central wavelengths of a line blend ( $\lambda \lambda$ ) is the weighted average of the NIST reported line centers.

${ }^{a}$ Reported velocities use FWHM for pure emission lines only, and actual ejecta velocity is FWHM/2. For P Cygni line profiles, the velocity is demarcated as either the blueshifted or redhifted component from rest wavelength. A negative equivalent width corresponds to an emission line.

b Blueshifted P Cygni component.

c Redshifted P Cygni component.

line widths probe the bulk ejecta velocity, because the lines may be formed in a wind.

Using the best-fit parameters, we estimate the blackbody luminosity of the photosphere. These luminosities are plotted in Figure 5 along with the Eddington luminosity for a $1.4 M_{\odot}$ WD, $L_{\text {Edd }}=1.75 \times 10^{38} \mathrm{erg} \mathrm{s}^{-1}$, calculated using a $100 \%$ ion- ized atmosphere and Thompson scattering opacity. On 2010 February 6.98, we find that $L=(2.71 \pm 0.22) \times 10^{39} \mathrm{erg} \mathrm{s}^{-1}$, and the luminosity declines by a factor of four over the next three days. SN 2010U is clearly super-Eddington for at least the four days near maximum light, in agreement with determination by Humphreys et al. (2010). 


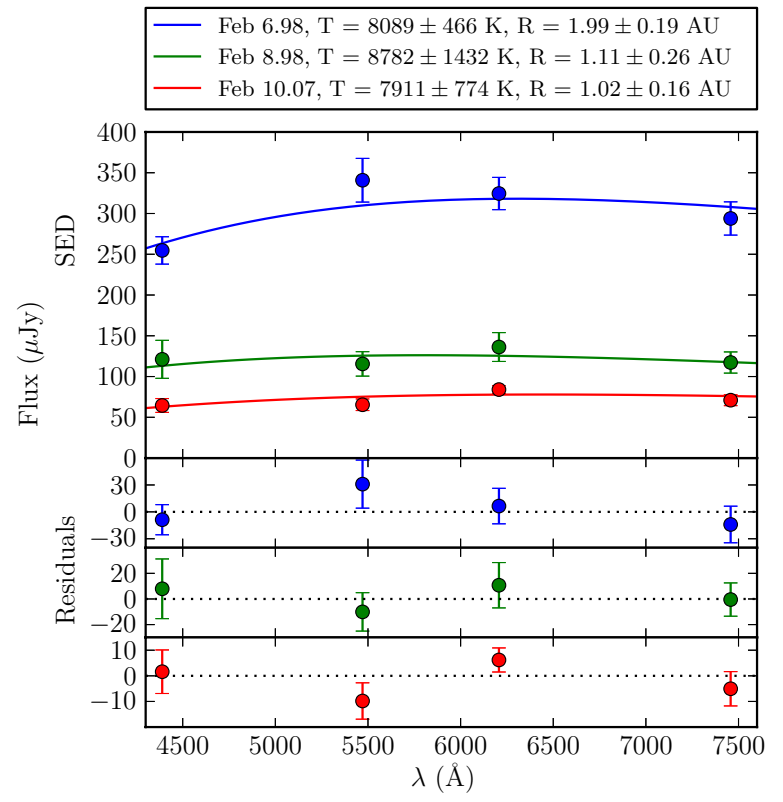

Figure 4. Best-fit radius and temperature parameters for each epoch. Within the errors, the temperature of the photosphere remains constant over the three epochs, while the radius of the photosphere recedes to $\approx 1.0 \mathrm{AU}$ as the envelope becomes optically thin.

(A color version of this figure is available in the online journal.)

The super-Eddington luminosity of SN 2010U is similar to that of L91, where model-atmosphere fitting to UV and optical data by Schwarz et al. (2001) determined that it remained superEddington from -5 days $<t<8$ days around maximum light. They find a peak bolometric flux of $L=(2.6 \pm 0.3) \times$ $10^{39} \mathrm{erg} \mathrm{s}^{-1}$ with $R=0.7 \mathrm{AU}$ and $T=1.3 \times 10^{4} \mathrm{~K}$ at maximum light. Schwarz et al. (2001) determine from their model that the radiative forces are 10 times the gravity forces for the entire atmosphere, thus the "atmosphere" should appear as a radiatively driven wind. Shaviv (2001) suggests that a clumpy but porous photosphere would enable steady-state super-Eddington luminosities to persist for an extended period.

\subsection{Spectroscopic Evolution}

Humphreys et al. (2010) published a spectrum of SN $2010 \mathrm{U}$ 14 days after maximum light, noting the presence of $\mathrm{H} \alpha, \mathrm{H} \beta$, and $\mathrm{O}_{\mathrm{I}} \lambda \lambda 7774$. They emphasize that this spectrum does not resemble that of a supernova nor any ILOTs such as SN $2008 \mathrm{~S}$ nor NGC 300 OT.

Our three epochs of spectroscopy trace the CN spectral evolution of SN 2010U from 1.03 days after maximum light (2010 February 7.30) to 15.30 days after maximum light (2010 February 21.51; Figure 6). The earliest spectrum exhibits strong emission lines of (in decreasing strength) the hydrogen Balmer series, Fe II, Na I, O I, N I, and C I. Spectra were de-redshifted to match the $[\mathrm{S} \mathrm{II}] \lambda 6716.44, \lambda 6730.82$ emission lines from the host galaxy $(z=0.00087)$. The radial velocity of SN $2010 \mathrm{U}$ is $-260 \mathrm{~km} \mathrm{~s}^{-1}$, while the NASA/IPAC Extragalactic Database redshift of NGC 4214 is $-290 \mathrm{~km} \mathrm{~s}^{-1}$. This $\Delta v \approx-30 \mathrm{~km} \mathrm{~s}^{-1}$ is consistent with the internal motions of the galaxy.

Strong P Cygni profiles are clearly seen in the Na I D $\lambda 5892$ and $\mathrm{O}_{\mathrm{I}} \lambda 7774$ lines (Figure 7). The presence of these profiles in the 2010 February 7.30 spectrum (Figure 6) are characteristic of spectra of novae at maximum light (Warner 2003), therefore with this additional information to the initial rise in $r^{\prime}$ band, it is likely that the light curve (Figure 2) captures the maximum light of SN 2010U. We take an average of the velocities of the

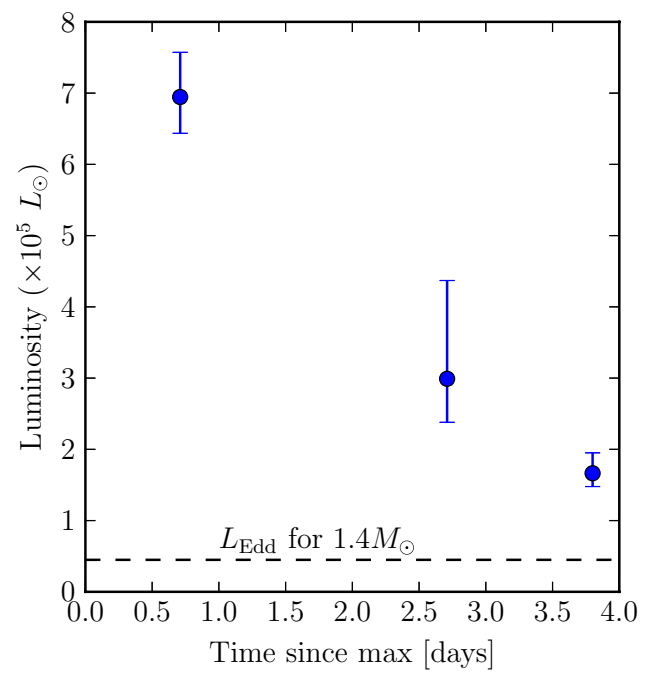

Figure 5. Bolometric luminosity of SN 2010U determined from fits of a spherical blackbody to photometry (see Figure 4). Shortly after maximum light (+0.71 days), we find that SN $2010 \mathrm{U}$ is clearly super-Eddington with luminosity $L=(2.71 \pm 0.22) \times 10^{39} \mathrm{erg} \mathrm{s}^{-1}$. Over the next three days, the luminosity declines by a factor of four.

(A color version of this figure is available in the online journal.)

P Cygni lines of Na I D and O I (Figure 7) and the widths of the Balmer series and $\mathrm{O}_{\mathrm{I}}$ (Figures 8 and 9) to derive an expansion velocity of $\approx 1100 \mathrm{~km} \mathrm{~s}^{-1}$ (Figure 10 ).

When the envelope is initially optically thick, the radiation is ionization bounded and neutral and low-ionization emission lines are formed. As the nova evolves, the ionizing radiation becomes progressively harder as the photosphere recedes to the surface of the hot WD and higher ionization states are seen. Williams (1990) determines that for electron number densities $N_{e} \gtrsim 10^{9} \mathrm{~cm}^{-3}$ the nova envelope is optically thick, while forbidden lines will appear once $N_{e} \lesssim 10^{7} \mathrm{~cm}^{-3}$.

By 5.94 days after peak (2010 February 12.21), the P Cygni profiles become pure emission while the Balmer and Fe II emission lines are still clearly visible. The wider wavelength range of the NOT spectrum reveals $\mathrm{Ca}$ II $\mathrm{H}$ and $\mathrm{K}$ emission lines and additional Balmer series lines continuing until the Balmer break. The most significant change is the increase in strength of the OI $\lambda 7773$ and $\lambda 8446$ lines and the appearance of the forbidden lines of [O I] $\lambda 5577,6300$, and 6363.

By 15.30 days after peak, the Fe II emission lines have mostly faded and the Balmer series dominates in emission (Figure 11). Throughout all spectral epochs, the Balmer lines are the strongest emission lines (Figure 8), evolving from an FWHM of $2200 \mathrm{~km} \mathrm{~s}^{-1}$ at 1.03 days after maximum light to $1600 \mathrm{~km} \mathrm{~s}^{-1}$ at 15.30 days after maximum light. Initially, the Balmer profiles show an asymmetric structure, but then evolve to become narrower and more symmetric. The O I $\lambda 8446$ line developed a flat-topped profile (Figure 9) characteristic of an optically thin expanding spherical shell at a velocity of $815 \mathrm{~km} \mathrm{~s}^{-1}$. There are also faint forbidden lines of [O I], signaling the entrance into the nebular phase of $\mathrm{CN}$ spectral evolution. The late time spectrum is characteristic of a nova shell, showing strong Balmer lines, O I, and signs of [O I] $\lambda 6300$, which signals the transition from the permitted state to the forbidden state.

Smith et al. (2011) argues that, based only upon an early spectrum from Keck/LRIS on 2010 February 7th UT (two days after maximum light), the spectra and light curve are very similar to an LBV, (SN) 2000ch (Wagner et al. 2004; Pastorello 


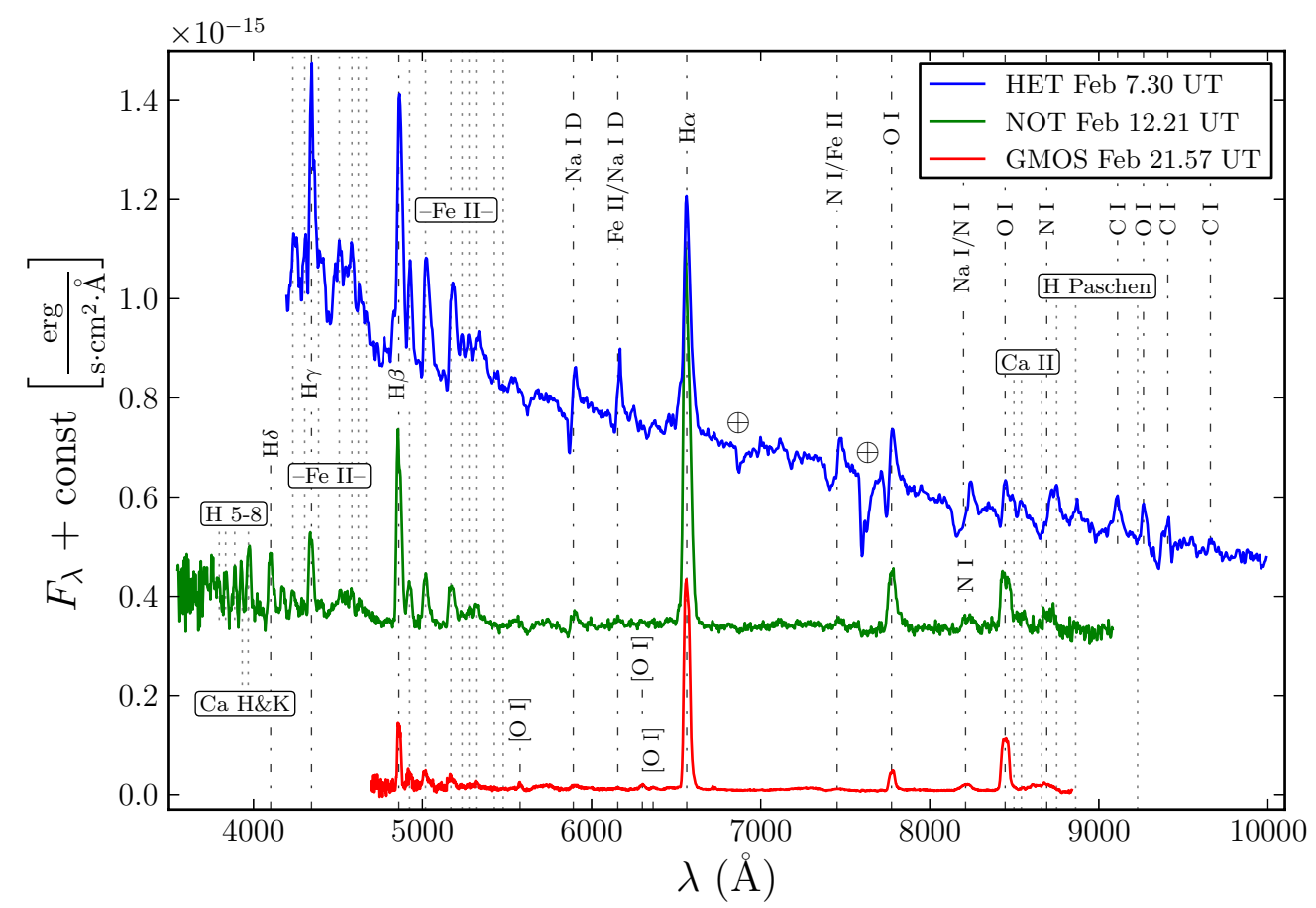

Figure 6. Multi-epoch spectra of SN 2010 U capturing the post-maximum "iron-curtain" stage (HET), "pre-nebular" stage (NOT), and the onset of the nebular stage (GMOS). Spectra were de-redshifted to match the [S II] $\lambda 6716.44, \lambda 6730.82$ emission lines from the host galaxy $(z=0.00087)$. Strong P Cygni profiles are present at early times (see Figure 7), but quickly fade into the continuum.

(A color version of this figure is available in the online journal.)

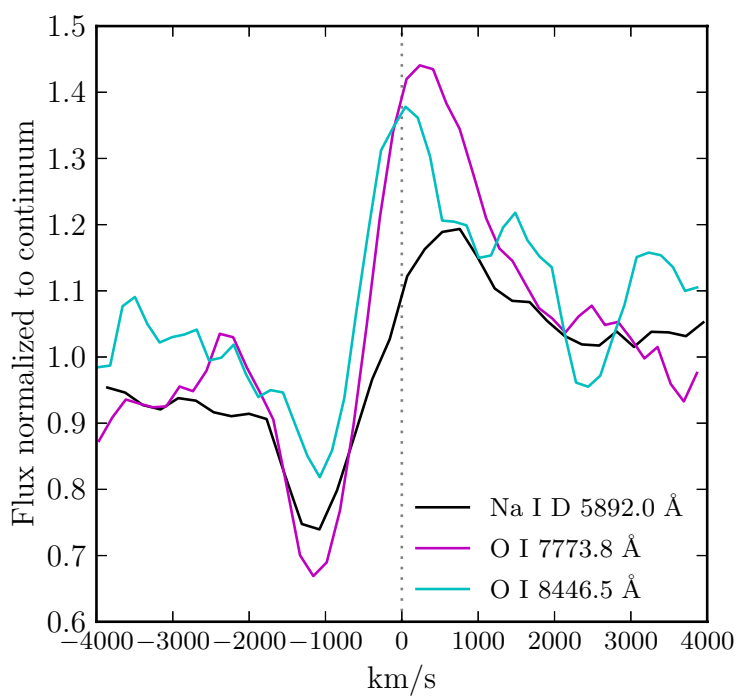

Figure 7. Na I D and O I P Cygni profiles from the 2010 February 7.30 spectrum, taken 1.03 days after maximum light. The P Cygni profiles trace the ejecta velocities from the nova explosion, since at early phases the ejecta is optically thick and approximates the true expansion velocity. Na I D and O I are doublet and triplet lines, respectively, and are centered relative to the weighted means of the NIST atomic line database relative strengths. The line flux is normalized to continuum.

(A color version of this figure is available in the online journal.)

et al. 2010). However, upon examination of extant nova spectra, a surprisingly close match to SN $2010 \mathrm{U}$ is found with L91 (Figure 13). The prominent Fe II and O I emission visible in the late time spectrum additionally suggests that SN $2010 \mathrm{U}$ is not an LBV because most known LBVs do not show this behavior (Smith et al. 2011).

Williams (1992) devises an optical spectroscopic classification system that categorizes novae by their strongest non-Balmer emission lines, typically $\mathrm{Fe}$ II or a combination of $\mathrm{He}$ and $\mathrm{N}$, called $\mathrm{He} / \mathrm{N}$. They find that $\mathrm{He} / \mathrm{N}$ novae preferentially have shorter $t_{2}$, higher expansion velocities, and coronal lines, while $\mathrm{Fe}$ II novae evolve to a forbidden line spectrum with lower ionization species. The $\mathrm{He} / \mathrm{N}$ spectrum is formed in a discrete shell ejected during the explosive thermonuclear runaway while the Fe II spectrum is formed in a continuous wind driven by the radiation from the residual burning of material on the surface of the WD. Williams (1992) explains that manifestation of the spectrum is dependent on which mechanism dominates in a two-component model. The spectral evolution of SN 2010U and its evolution clearly identifies it as a member of the Fe II spectral class.

We observe velocity evolution in the emission line profiles of SN 2010U (Figure 10), with the Balmer and O I $\lambda 7774$ and $\lambda 8446$ profiles exhibiting a jagged shape at early times, and then becoming smoother and narrower. For Fe II novae, this is the result of a photosphere formed in a wind with velocity homologously increasing outward and mass-loss rate decreasing with time. The decreasing density pushes the region of line formation steadily inward toward the surface of the WD where flow velocities are lower.

Schwarz et al. (2011) find that the presence of [Fe x] 6375 requires a hot photoionization source, and thus correlates well with Super Soft X-ray emission. That this line is not visible in the nebular spectra complements the non-detection of X-ray emission from SN 2010U.

\section{SN 2010U AS A FAST AND EXTREMELY LUMINOUS CLASSICAL NOVA}

\subsection{Comparison to Nova LMC 1991 and M31N-2007-11d}

Although roughly a thousand $\mathrm{CNe}$ have been discovered, only a few luminous events are known due to their rapid decline 


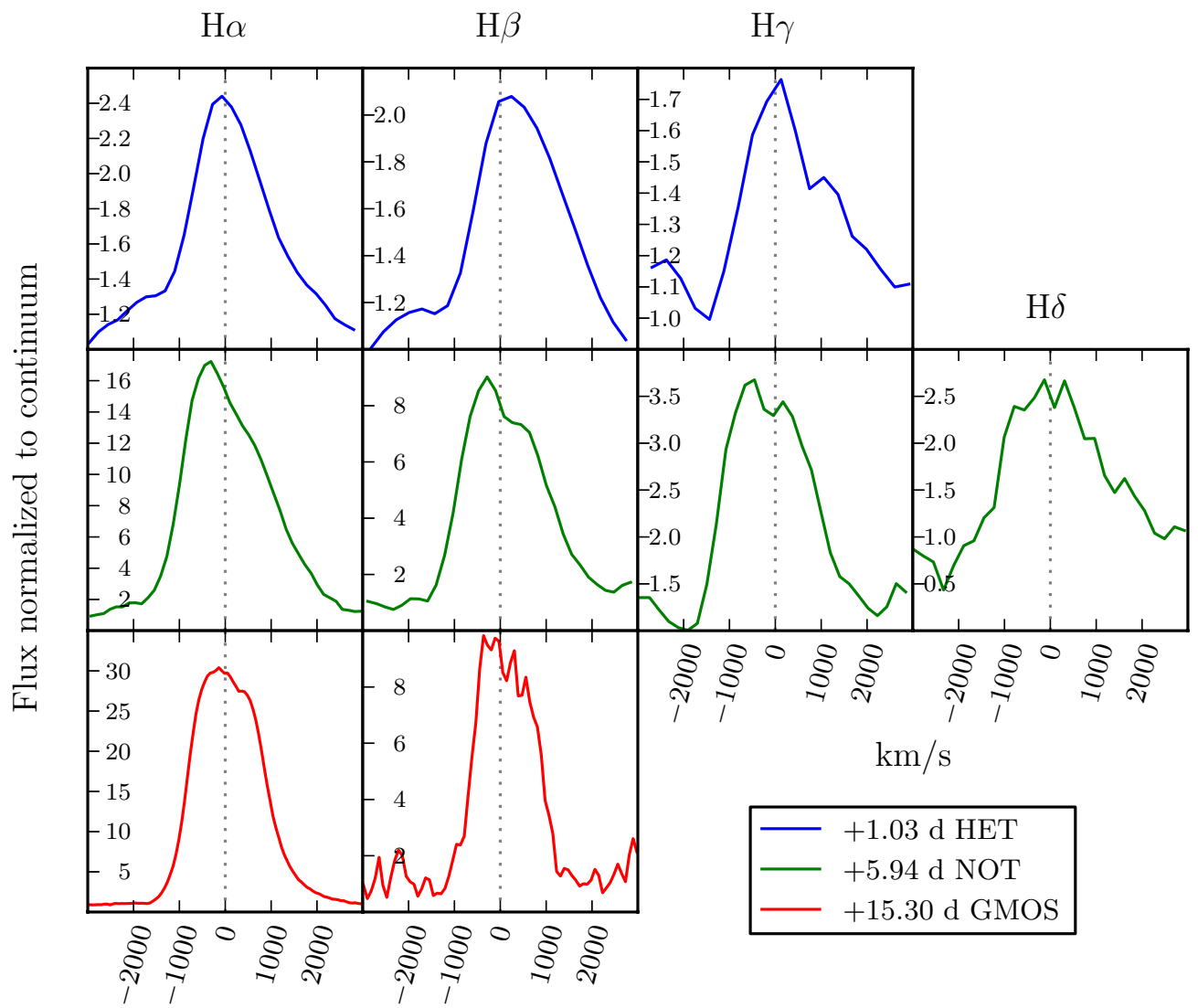

Figure 8. Hydrogen Balmer line profiles for SN 2010U. The profiles become narrower and more box-like with time, and the FWHM decreases with time. The line profiles are scaled relative to the continuum flux.

(A color version of this figure is available in the online journal.)

O I $7773.8 \AA \quad$ O I $8446.5 \AA$

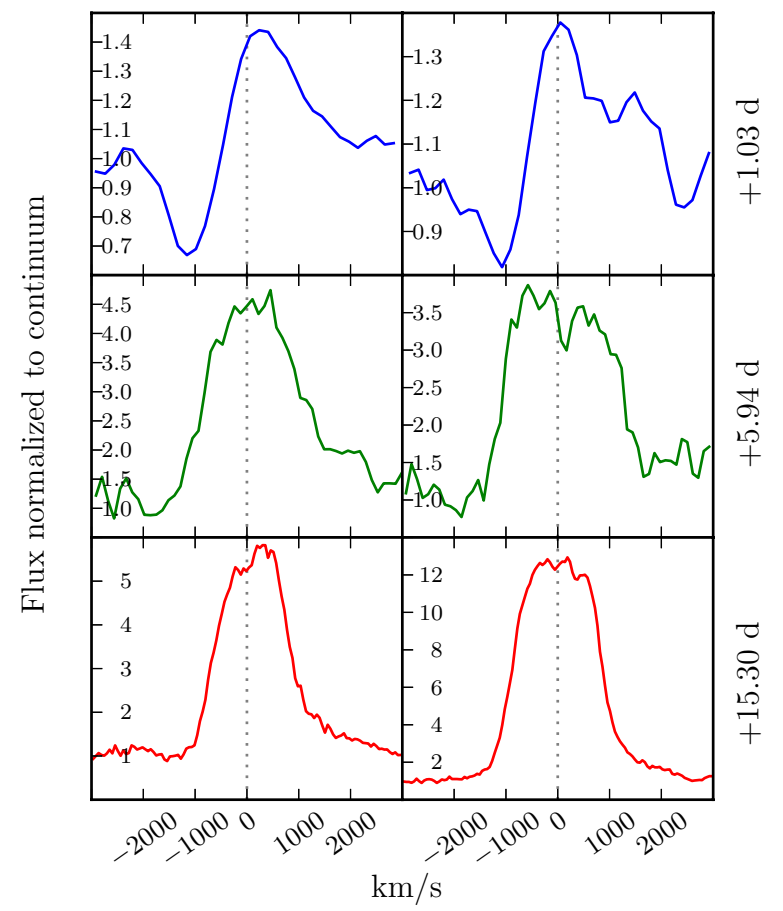

Figure 9. O I $\lambda 7774$ and $\lambda 8446$ line profiles. Initially, these are P Cygni line profiles characteristic of an optically thick expanding envelope that develop into a flat-topped profile, characteristic of an optically thin expanding shell. Flux is normalized to the continuum.

(A color version of this figure is available in the online journal.)

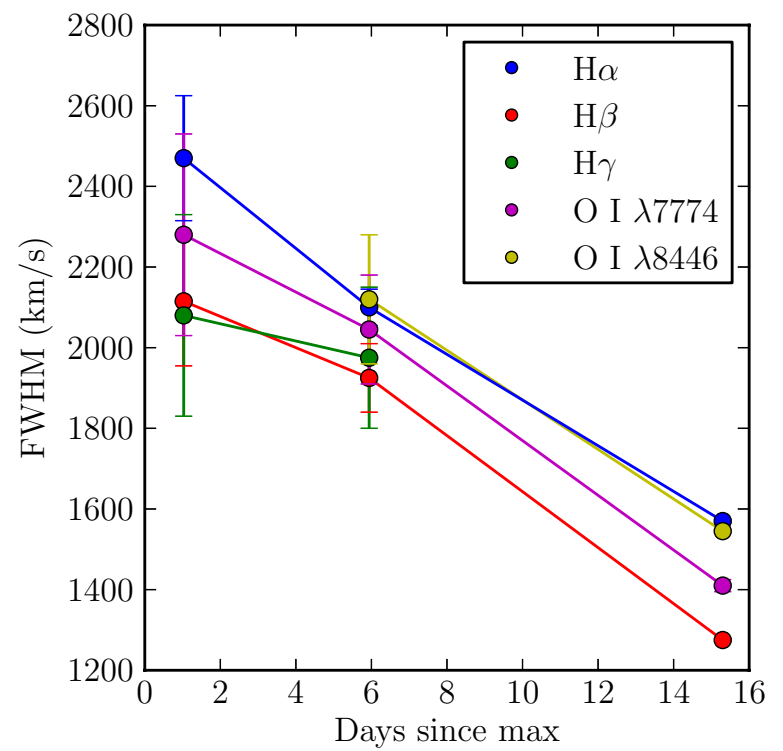

Figure 10. All velocities plotted are FWHM except the $t=0$ day O I points, which are derived from the P Cygni profiles. For compatibility with this plot, line species with P Cygni profiles (OI) are plotted as twice the velocity of the blueshifted absorption component. The FWHM of the lines decreases with time. (A color version of this figure is available in the online journal.)

and intrinsic rarity. Two other luminous Fe II type novae have been studied extensively: L91 (Della Valle 1991; Schwarz et al. 2001; Williams et al. 1994) and M31N-2007-11d (Shafter et al. 2009), hereafter M31N. L91 was an exceedingly bright and fast Fe II type nova in the Large Magellanic Cloud, so luminous 


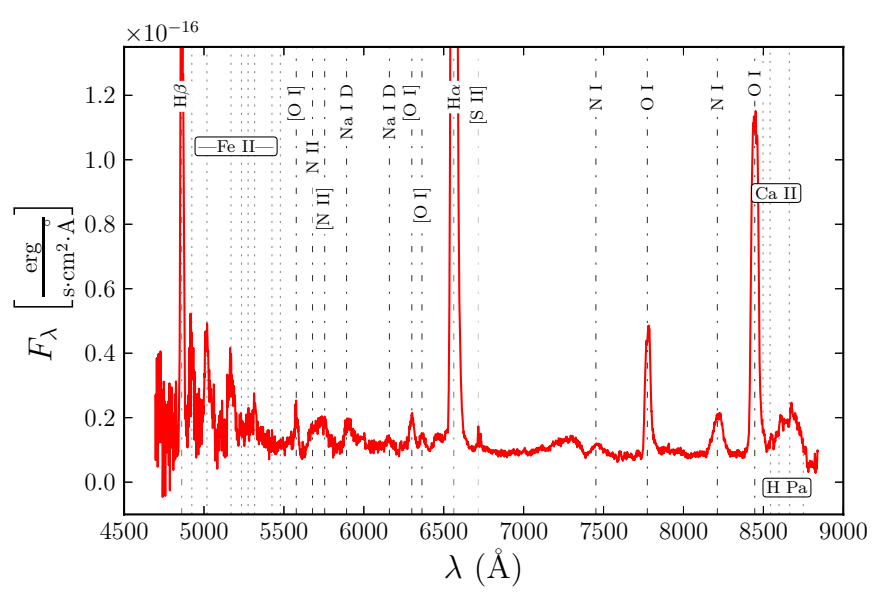

Figure 11. Late time (15.3 days after maximum) spectrum of SN 2010U. The strong presence of $\mathrm{O}_{\mathrm{I}}$ and the first appearance of [O I ] emission suggests that SN 2010U is entering the nebular stage. Host galaxy lines (such as [S II]) are labeled in gray, while multiplet species are labeled with dotted lines.

(A color version of this figure is available in the online journal.)

that it was initially heralded as a prototype for a class of superbright novae (Della Valle 1991). M31N was discovered during a spectroscopic survey of novae in M31 by Shafter et al. (2011).

The light curves of L91 and M31N are similar to SN 2010U (a comparison between SN 2010U and L91 is shown in Figure 12). L91 was discovered five days before maximum light and $\mathrm{M} 31 \mathrm{~N} \gtrsim 4$ days before maximum. The rise to maximum of L91 is among the longest for novae on record, with a peak of $M_{v}=-10.0 \mathrm{mag}$. The light curve of L91 shown here is drawn from the photometry published in the circulars (Shore et al. 1991; Gilmore 1991; Gilmore et al. 1991; Liller et al. 1991; Della Valle et al. 1991). Shafter et al. (2009) set a lower limit of four days on the rise time for $\mathrm{M} 31 \mathrm{~N}$ from quiescence to a maximum light of $M_{V} \simeq-9.5 \mathrm{mag}$. Both novae declined rapidly from maximum light with $t_{2}=6 \pm 1$ days (L91; Schwarz et al. 2001) and $t_{2}=9.5$ days (M31N; Shafter et al. 2009). By comparison, SN 2010U has $t_{2}=3.5 \pm 0.3$ days and the rise time is unconstrained.

Spectroscopically, L91 and M31N are remarkably similar to SN 2010U-they are all clearly Fe II type novae. L91 and M31N have slightly lower expansion velocities with $\mathrm{H} \alpha$ FWHM of $\simeq 1880 \mathrm{~km} \mathrm{~s}^{-1}$ and $\simeq 1550 \mathrm{~km} \mathrm{~s}^{-1}$, respectively, while SN $2010 \mathrm{U}$ has $\simeq 2230 \mathrm{~km} \mathrm{~s}^{-1}$. At early times both L91 and M31N show strong P Cygni absorption profiles. L91 clearly mirrors the temporal and spectral evolution of SN 2010U (Figure 13).

L91 is one of the best-studied novae of the modern era. UV spectra from the IUE satellite revealed strong Fe II absorption, which would be reradiated as emission in the optical (Schwarz et al. 2001). Schwarz et al. (2001) construct a model atmosphere of L91 using PHOENIX and CLOUDY to obtain abundance estimates of the outburst and find that L91 was enriched in CNO elements and originated from a carbon-oxygen (CO) WD.

Although there is no late time spectroscopy of SN 2010U, the spectrum 15.94 days after maximum light already shows evidence of forbidden oxygen, with no evidence for any neon. This, combined with the presence of carbon and oxygen and the similarity of spectra to L91, suggests that SN 2010U also had a CO WD progenitor.

However, efforts to identify WD progenitor types are confounded by the possibility that an enriched envelope could exist on top of a $\mathrm{CO} \mathrm{WD}$, or that an $\mathrm{ONeMg}$ nova may or may not

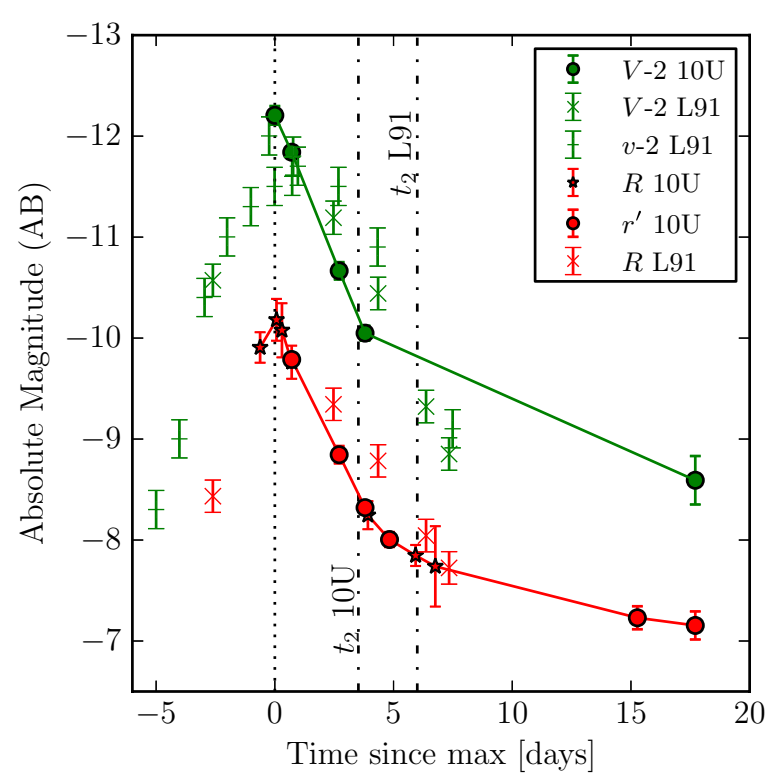

Figure 12. Comparison of SN $2010 \mathrm{U}$ (10U) to L91. " $v$ " represents visual magnitude, and $v$-band and $V$-band measurements are offset by two magnitudes for clarity. Both novae exhibit similar peak absolute brightnesses and decline rates. The rise time of L91 (and M31N) is exceptionally long, while the rise time of $10 \mathrm{U}$ is unconstrained.

(A color version of this figure is available in the online journal.)

have a dredge-up event that would enrich the spectrum, producing a wide range of observable spectra. Whether or not there is a direct mapping between the manifestation of the spectrum and the composition of the underlying WD is still an open question (Prialnik \& Kovetz 1998; Mason 2011), although with detailed UV and X-ray spectral observations capturing the entirety of the nova outburst, such as in the case of L91 (Schwarz et al. 2001), it may be possible to tell.

\subsection{MMRD and FWHM Relationships}

Studies of novae have revealed a correlation between peak absolute magnitude $M_{V}$ and decline rate $t_{2}$, termed the maximum magnitude versus rate of decline relationship (MMRD). The shorter $t_{2}$, the more intrinsically luminous the nova explosion (Della Valle \& Livio 1995; Downes \& Duerbeck 2000; Shafter et al. 2011).

Shafter et al. (2011) executed an extensive multi-year study of novae in M31, discovering and spectroscopically classifying 46 novae, bringing the total of spectroscopically classified novae in M31 to 91. They derive an MMRD for M31 novae and compare to other historical samples of novae (Figure 14; Della Valle \& Livio 1995; Downes \& Duerbeck 2000; Shafter et al. 2011). There is a substantial amount of scatter in this relation. The extreme quadrant of the MMRD at high luminosities and shortest $t_{2}$ is shown in Figure 15, with the most luminous novae known to date compiled by Shafter et al. (2009) and updated with recent discoveries by Kasliwal et al. (2011) and Shafter et al. (2011). Although in Figure 15 there appear to be a comparable number of luminous $\mathrm{He} / \mathrm{N}$ novae and $\mathrm{Fe}$ II novae, because $\mathrm{He} / \mathrm{N}$ novae are rarer they are in fact preferentially brighter and faster than Fe II novae (Williams 1992; Shafter et al. 2011, 2012). For example, Shafter et al. (2011) find in their M31 survey that three of their four fastest declining novae are $\mathrm{He} / \mathrm{N}$ type, although by number $\mathrm{He} / \mathrm{N}$ novae comprise only $\sim 20 \%$ of all novae.

Despite claims that there might be a "super-bright" class of novae (Della Valle 1991), Shafter et al. (2009) find no evidence 


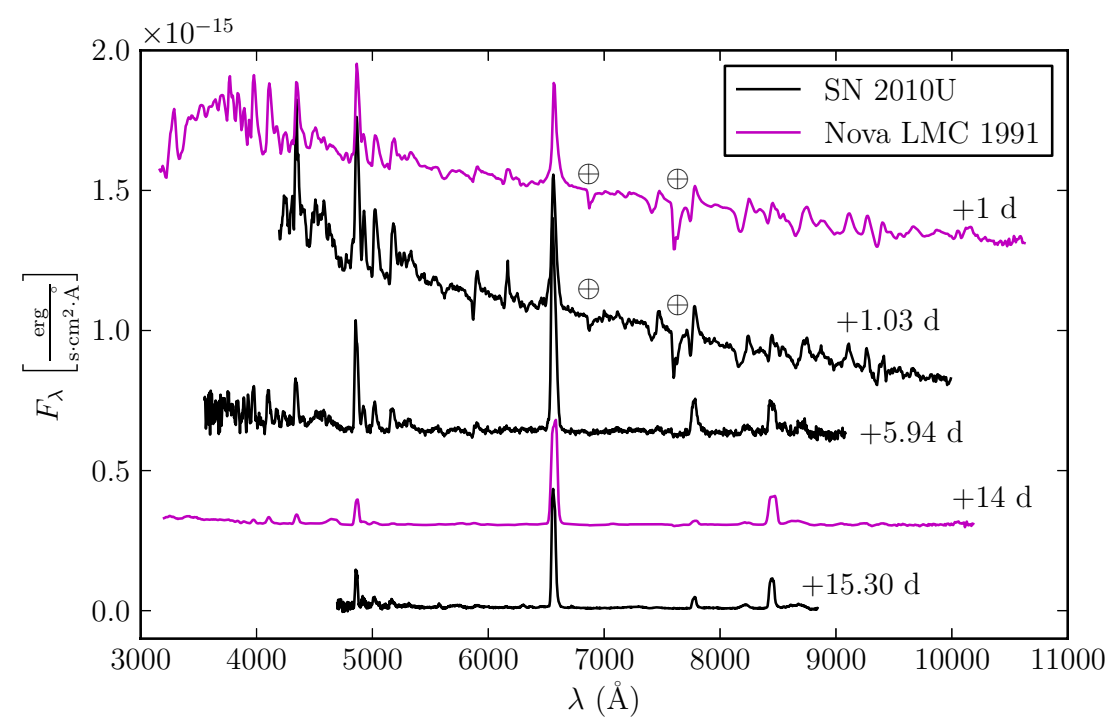

Figure 13. Spectroscopic comparison to L91. Both novae exhibit strong Fe II lines, hydrogen Balmer profiles with similar widths, P Cygni profiles at early times, and similar timescales for spectral evolution.

(A color version of this figure is available in the online journal.)

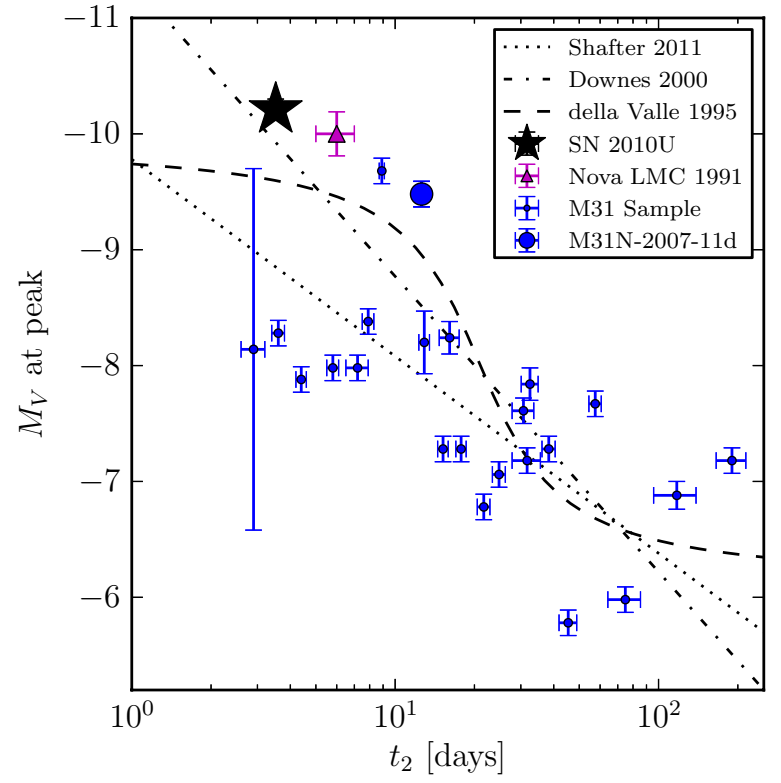

Figure 14. Maximum magnitude vs. rate of decline (MMRD) relationship created from data from the Shafter et al. (2011) survey with luminous novae and historical MMRD relationships overplotted.

(A color version of this figure is available in the online journal.)

for a distinct population. However, SN 2010U is indeed a very luminous nova: Compared with the 883 novae on record compiled by Shafter et al. (2009), only four novae are brighter, two of which are not spectroscopically confirmed (Ciardullo et al. 1987; Kasliwal et al. 2011). At the extreme end of the luminosity distribution, there is large scatter from observational uncertainties such as intrinsic extinction and uncertainty in the capture of maximum light as well as uncertainties from intrinsic variability in the novae explosion due to variation in WD mass, accretion rate, and metallicity (Shafter et al. 2009).

Surveys also find that novae with faster expansion velocities have a faster decline from maximum light (Figure 16) (McLaughlin 1960; Shafter et al. 2011). The scatter in Figure 16 is likely due to the time dependence of velocities, which depends on how soon after maximum light the spectra was obtained. Such a $t_{2}$ versus $\mathrm{H} \alpha$ FWHM relationship is a natural outcome if $\mathrm{He} / \mathrm{N}$ novae are the fastest declining and the most violent, having the highest ejecta velocities. SN $2010 \mathrm{U}$ is a fast declining nova with a moderate ejection velocity.

\subsection{Nova Populations and Progenitor}

Since the spectroscopic classification scheme of Williams et al. (1991) was devised and the MMRD predicted that more massive WDs produce more violent explosions (see Section 4.4; Della Valle \& Livio 1995), an outstanding question has been whether the spectral type of a nova correlates with the properties of the underlying stellar population. Extragalactic studies have revealed that, in general, the nova population follows the galaxy light (Ciardullo et al. 1987; Capaccioli et al. 1989; Shafter \& Irby 2001; Shafter et al. 2012). Surveys of multiple galaxies of different morphologies aim to determine whether novae properties such as peak brightness, $t_{2}$, and spectral type correlate with the underlying stellar population.

In the Milky Way, Della Valle \& Livio (1998) find that $\mathrm{He} / \mathrm{N}$ novae are concentrated at the Galactic plane and are fast and bright, while $\mathrm{Fe}$ II novae are concentrated in the bulge and thick disk of the galaxy and are slow and dim. Because younger stellar populations have on average more massive WDs, disk novae should be more extreme; therefore they claim that $\mathrm{He} / \mathrm{N}$ novae are associated with a younger stellar population and that the Fe II novae are associated with an older stellar population.

In M31, Shafter et al. (2011) find conflicting results with no compelling evidence that spectroscopic class is a strong function of galactic location, although the spatial distribution $\mathrm{He} / \mathrm{N}$ novae is slightly more extended than that of Fe II novae. They also find that the spatial distribution of faster (smaller $t_{2}$ ) novae is slightly more extended than that of slower novae. In their X-ray survey of M31 novae (see Section 3.2), Henze et al. (2011) find that novae with longer turn-on times (implying more massive ejecta and thus a less massive WD) are more centrally concentrated in the bulge while those with shorter turn-on times are preferentially located in the disk, agreeing with the interpretation of Della Valle \& Livio (1998) and the 


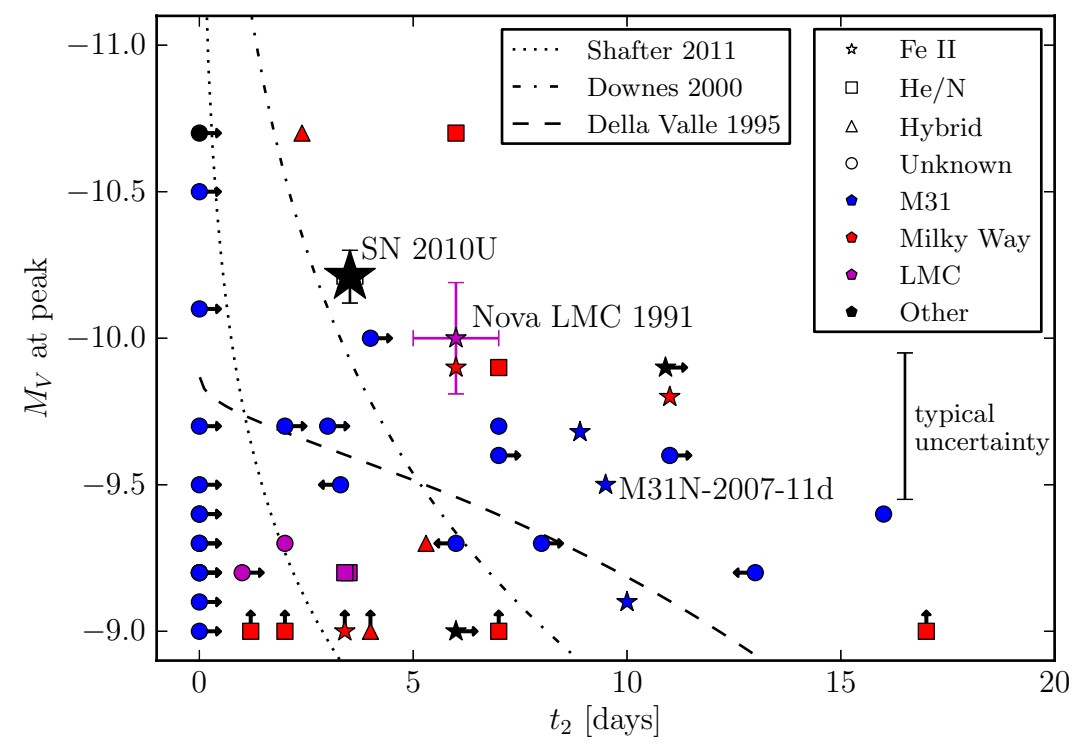

Figure 15. MMRD relationships (Della Valle \& Livio 1995; Downes \& Duerbeck 2000; Shafter et al. 2011) plotted over the most luminous novae on record Shafter et al. (2009), Kasliwal et al. (2011), and Shafter et al. (2011). Upper or lower limits on $t_{2}$ are shown with arrows, and novae for which no $t_{2}$ measurement exists are plotted at $t_{2}=0$. The colors denote location and the symbols denote spectral type. All magnitudes are $V$ band except for the black points other than SN 2010U, which are measured in $g^{\prime}$ (Kasliwal et al. 2011). The typical uncertainty is shown, which is largely an uncertainty of whether the nova was caught at peak magnitude.

(A color version of this figure is available in the online journal.)

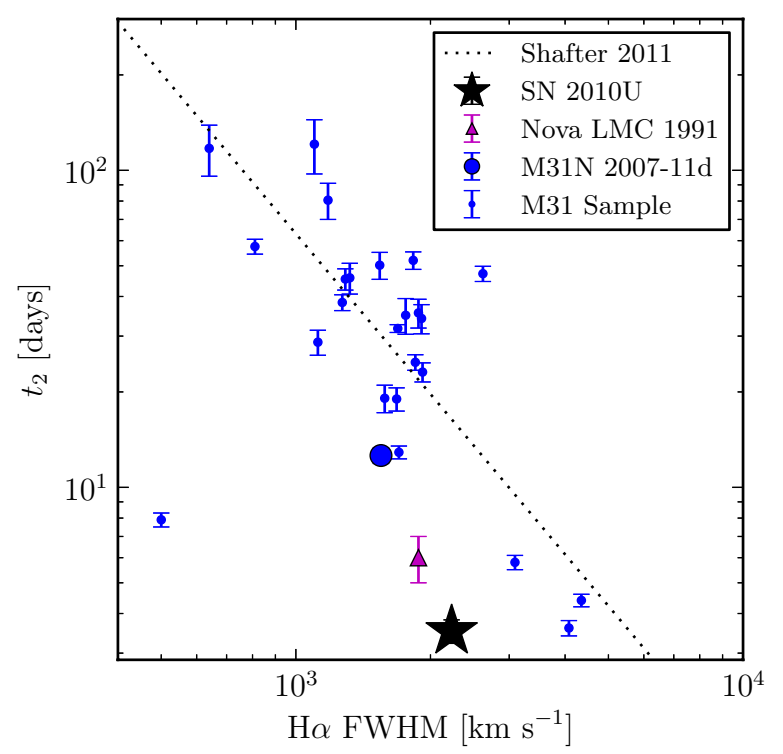

Figure 16. H $\alpha$ width compared to decline speed (measured by $t_{2}$ ) of the nova outburst for the Shafter et al. (2011) sample of novae from M31, with L91 and SN 2010U also plotted. SN 2010 U and L91 are at slightly slower velocities than the mean, but still consistent with the trend, given the large scatter. Interestingly, the three bright Fe II novae are all faster declining than the mean.

(A color version of this figure is available in the online journal.)

Shafter et al. (2011) results that novae with higher mass WDs are found in the disk.

In M33, however, Shafter et al. (2012) find that five of eight novae are $\mathrm{He} / \mathrm{N}$ or $\mathrm{Fe}$ IIb hybrid novae, while only two are definitively of the Fe II class. Hybrid novae initially have broad Fe II lines, which then later are replaced by $\mathrm{He} / \mathrm{N}$ lines. Interestingly, the opposite evolution from $\mathrm{He} / \mathrm{N}$ to $\mathrm{Fe}$ II has never been observed. They speculate that this statistically significant difference in the fraction of $\mathrm{He} / \mathrm{N}$ to $\mathrm{Fe}$ II Galactic and M31 novae could be a result of the underlying population of M33, which is a bulgeless galaxy, and therefore would be expected to be dominated by a disk population. These spectroscopic results of Shafter et al. (2012) confirm the results of a photometric study of M33 novae by della Valle et al. (1994), which claimed that because M33 novae were intrinsically brighter and recurred more frequently than those in bulgedominate systems, novae in disk-dominated systems are likely associated with more massive WDs.

To address whether or not the most luminous Fe II novae are associated with a particular stellar population, it is useful to investigate the associated stellar populations of L91, M31N, and SN 2010U. Subramaniam \& Anupama (2002) examine the region surrounding L91 and find that there are three clusters within $\sim 130 \mathrm{pc}$ with ages less than the young age of $10^{7.5} \mathrm{yr}$, and that the location of L91 is close to another fast nova, LMC 1977\#2. Because the LMC is a bulgeless galaxy, we would expect it to be dominated by fast declining novae, and indeed it possesses a fast declining and fast ejecta population of novae (Della Valle \& Duerbeck 1993). However, that the luminous Fe II nova L91 specifically came from a young stellar population is in tension with the prediction that Fe II novae are associated with older stellar populations.

The location of M31N-2007-11d is at large galactocentric radius from the center of M31 and perhaps a member of the disk, although another luminous but less-studied Fe II nova M31N-2009-09b is close to the center of the galaxy. However, the large inclination of M31 makes it difficult to determine whether or not M31N-2009-09b is actually in the bulge or might be within the disk and projected in front of the bulge.

Humphreys et al. (2010) use pre-explosion archival Hubble Space Telescope images with WFC3 F814W and WFPC2 F555W and F814W to investigate the progenitor of SN 2010U and the associated stellar population. At the location of the nova, there is a photometric limit of $M_{V} \approx-3.2 \mathrm{mag}$, which puts an upper limit on the mass of the progenitor system and its companion of $3-5 M_{\odot}$. The stars within $100 \mathrm{pc}$ of the location of the nova are dim and red, suggesting association with an evolved population. The spatial location of any younger main-sequence stars is distinct from the location of the nova, suggesting it is not associated with a massive star population, although it could 
be an evolved and obscured lower mass asymptotic giant branch star (Humphreys et al. 2010). If SN 2010U originated from an evolved population, this would follow the emerging trend that Fe II novae come from lower mass, older populations such as the bulge of M31.

Based upon the theory of the MMRD (see Section 4.4), the high intrinsic luminosity and fast temporal evolution of SN 2010U signal that the progenitor was a high-mass WD. The evolutionary channels of how the binary system could reach its pre-outburst state depend on when the WD was born. The WD could be born massive from a massive star, or alternatively the WD could have formed from a less massive star and accreted material. If the WD was born massive, there is a higher chance that it would be an ONeMg WD. Some claim that "neon novae," which exhibit strong [ $\mathrm{Ne}$ III] and [ $\mathrm{Ne} \mathrm{V}]$ lines during the forbidden phase, and thus have a high neon abundance, originate from ONeMg WDs (Mason 2011), although it is unclear whether or not these lines could be produced by an enriched surface layer and be uncorrelated with WD composition (Prialnik \& Kovetz 1998). The nova models of Yaron et al. (2005) are able to produce the full range of observed nova characteristics $\left(M_{V}\right.$, $t_{2}, v_{\text {ejecta }}$ ) using only $\mathrm{CO}$ WD progenitors, however with $\mathrm{ONeMg}$ WD progenitors they were able to produce ejecta envelopes that were enriched in neon by $\sim 10^{4}$ times compared with CO WDs.

Surveys have established that most luminous and fast novae are $\mathrm{He} / \mathrm{N}$ novae, however, SN 2010U, L91, and M31N are all Fe II novae. Shafter et al. (2009) speculate that what may set these novae apart is their long rise time compared to the general nova population, which reaches maximum light in less than three days. Schwarz et al. (2001) hypothesize that the long rise time is indicative of a large amount of ejected mass, such that the photons take a long time to diffuse and escape. For L91, Schwarz et al. (2001) found $M_{\mathrm{ej}} \sim 3 \times 10^{-4} M_{\odot}$ with a progenitor of a high-mass, cool WD with a low-metallicity envelope. Shafter et al. (2009) speculate that a long rise time may be related to the formation of the Fe II spectrum, which is formed in an optically thick wind driven by residual burning on the surface of the WD.

\subsection{Nova Physics and Super-Eddington Luminosity}

Della Valle \& Livio (1995) proposed that the MMRD is primarily a function of the mass of the WD progenitor. If the WD is more massive, the surface layers will be more degenerate and allow a more intense but also more rapid expulsion of material. Recent studies suggest that the outburst properties additionally depend sensitively upon other parameters of the progenitor system such as the temperature of the isothermal core and the accretion rate of material onto the surface of the WD. Townsley \& Bildsten (2005) calculate the ignition mass of the accreted material on the surface of the WD, $M_{\text {ign }}$, and its dependence on the mass transfer rate $\dot{M}$ to the WD, and the WD mass. The temperature of the WD core, $T_{c}$, also influences $M_{\text {ign }}$; however, Townsley \& Bildsten (2004) find that $T_{c}$ is set uniquely by $\dot{M}$, and therefore $M_{\text {ign }}$ is primarily a function of only two parameters. A lower $\dot{M} \lesssim 10^{-10} M_{\odot} \mathrm{yr}^{-1}$ leads to a lower $T_{c}$, which then increases the $M_{\text {ign }}$ needed to start the nova eruption. Alternatively, a high $\dot{M} \gtrsim 10^{-9} M_{\odot} \mathrm{yr}^{-1}$ will trigger the thermonuclear runaway earlier; at extremely high $\dot{M}$, stable hydrogen burning can occur (Townsley \& Bildsten 2004).

Yaron et al. (2005) show that very low accretion rates can produce the most extreme nova explosions, which are characterized by super-Eddington luminosities at maximum, large ejecta velocities, fast optical decline, and if the WD is of moderate mass, large ejecta masses. The extreme luminosities and rapid photometric declines of L91, M31N, and SN 2010U suggest that these novae all originated from massive WDs. Schwarz et al. (2001) claim that L91 had a $\gtrsim 1.2 M_{\odot}$ WD. Comparing the outburst characteristics ( $t_{2}$, bolometric luminosity, and ejecta velocity) of SN 2010U to the grid of theoretical predictions made by Yaron et al. (2005), we find that only the models with massive $\left(M \approx 1.25 M_{\odot}\right)$ and $\operatorname{cool}\left(T=3 \times 10^{7} \mathrm{~K}\right)$ WDs accreting at a very low rate $\left(\dot{M} \lesssim 10^{-11} M_{\odot} \mathrm{yr}^{-1}\right)$ are able to reproduce these parameters.

Schwarz et al. (2001) speculate that the large luminosity of L91, which would otherwise be inconsistent with the large ejecta mass, could be the result of a traveling shock wave through colliding ejecta shells. As described in Williams (1992), there is a discrete low-density and high-velocity shell and an optically thick wind which is powered by nuclear burning of residual material on the surface of the WD. He/N spectra are dominated by the discrete component, while Fe II novae are dominated by the wind component. A more massive ejecta shell would be more likely to have residual material which to burn on the surface of the WD, and may explain why the massive ejecta of L91 and likely massive ejecta of SN 2010U result in Fe II novae. Alternatively, common envelope interactions may be important for very luminous nova explosions. The outward moving nova shell quickly engulfs the secondary star, resulting in a stage of common envelope which can persist for several months after eruption. Iben \& Livio (1993) speculate that processes related to the common envelope, such as extra energy deposited by frictional drag can enhance rapid mass loss in super-Eddington novae.

The rise times of novae are generally longer than that of those predicted by spherically symmetric models, which suggests that time might be needed for the local thermonuclear runaway to proceed over the surface of the WD (Warner 2003), and may result in an asymmetric outburst. Williams et al. (1991) observe transient absorption features in high-resolution line profiles of Balmer and He I lines in other novae that are likely due to discrete absorption components, such as a small cloud of high density passing in front of the continuum source, suggesting the outburst is inhomogeneous. Clumpy ejecta would increase the effective Eddington limit and allow the nova outburst to sustain apparently super-Eddington luminosities for a period of time (Shaviv 2001). Given that nova ejecta are inhomogeneous and quickly evolving with time, it is likely that the super-Eddington novae L91 and SN 2010U sustained their remarkable luminosities through a porous photosphere or asymmetric explosion.

Although $\mathrm{He} / \mathrm{N}$ novae are preferentially brighter than Fe II novae, there are several very luminous Fe II novae, in particular L91, M31N, and SN 2010U. Although the MMRD and population studies suggest that Fe II novae should predominately come from older stellar populations which have on average smaller mass WDs, this mapping between WD and spectral type must not necessarily be direct. When a binary stellar system will evolve to a configuration that can produce a nova outburst is not a simple function of the mass or age of the dwarf, but is dependent upon the orbital parameters and stellar evolution of the binary system itself, for example, when the orbit might decay or the donor might evolve to fill its Roche lobe and begin mass transfer. Rather than originating from a fundamentally different population of progenitors, bright Fe II novae like L91, M31N, and SN 2010U likely represent novae drawn from an extreme corner of the parameter space that governs explosion physics: 
from binary systems with cool, high-mass CO WDs accreting material from their companions at a very low rate.

\section{CONCLUSIONS}

SN 2010U was a luminous (peak $M_{V}=-10.2 \pm 0.1 \mathrm{mag}$ ) and fast declining ( $t_{2}=3.5 \pm 0.3$ days) $\mathrm{CN}$ in the galaxy NGC 4214. Optical spectroscopy revealed that it was an Fe II type nova with strong hydrogen Balmer emission and expansion velocities of order $\approx 1100 \mathrm{~km} \mathrm{~s}^{-1}$. P Cygni spectral line profiles in spectra taken near maximum light indicate that the emission was optically thick and early photometry indicates that the optical emission was approximately thermal blackbody with $T \approx 8000 \mathrm{~K}$. As the nova faded, the spectrum evolved to a nebular state dominated by emission lines and [O I] emission began to appear.

Our conclusions are the following.

1. SN 2010U was a fast and luminous nova, among the top $0.5 \%$ brightest of all historical outbursts and the third brightest nova for which spectroscopic information exists. It is remarkably similar to both Nova LMC 1991 (L91) and M31N-2007-11d (M31N) in photometry and spectra.

2. SN $2010 \mathrm{U}$ is an Fe II type nova. Fe II novae are characteristically dimmer and slower to decline (longer $t_{2}$ ) than $\mathrm{He} / \mathrm{N}$. The existence of bright and fast Fe II novae like SN 2010U, L91, and M31N are interesting outliers in trends that aim to correlate spectral type with outburst properties.

3. SN 2010U reached super-Eddington luminosities during the peak of its outburst. Most novae are sub-Eddington, however L91 was also super-Eddington for an extended period of time while it ejected a large amount of mass. It is likely that the Fe II spectrum, which is formed in an optically thick wind, is related to high mass loss.

4. Massive and luminous nova outbursts like SN 2010U probe a unique set of progenitor parameters, and point to an extreme region of parameter space with low accretion rate, high WD mass, and low WD core temperature. The extreme luminosity region of the MMRD is poorly constrained and subject to high scatter.

5. That SN 2010U likely originated from a CO WD associated with an evolved stellar population is interesting in the context of the debate of nova populations and the manifestation of nova spectral type. Trends in the Milky Way, the LMC, and M33 suggest that more luminous novae of the $\mathrm{He} / \mathrm{N}$ type originate from young stellar populations where average WD mass is higher. However, L91, M31N, and SN $2010 \mathrm{U}$ all are extremely luminous Fe II novae that are likely from massive CO WDs. Various paths of binary evolution can influence when these systems will enter a configuration that would generate nova outbursts.

Upcoming wide-field transient surveys like LSST will discover optical transients in ever greater numbers. In particular, the high cadence and deep optical limits of the survey will reveal many $\mathrm{CNe}$, which have traditionally been difficult to study because of moderate luminosities and fast decline from maximum light. Understanding the extreme quadrant of high luminosity and rapid optical decline for $\mathrm{CNe}$ is paramount for maximizing the scientific return of large photometric surveys, for which spectroscopic resources will not be available to confirm every discovery. Accurately characterizing the intermediate luminosity phase space now will be paramount to understanding the wealth of data from future transient surveys.
I.C. thanks Greg Schwarz, Robert Williams, Maxwell Moe, Alica Soderberg, Warren Brown, James Moran, and Rosanne Di Stefano for valuable conversations about nova physics, and Wen-fai Fong for helpful comments on this manuscript. We graciously acknowledge the amateur astronomers who contributed critical discovery images: K. Itagaki, J. Brimacombe, T. Yusa, and J. Nicolas. I.C. is supported by the NSF Graduate Research Fellowship Grant. J.C.W.'s group at UT Austin is supported by NSF Grant AST 11-09881. J.V. is supported by Hungarian OTKA Grant K76816. This research was completed through the use of many facilities. It is based on observations made with the Nordic Optical Telescope, operated on the island of La Palma jointly by Denmark, Finland, Iceland, Norway, and Sweden, in the Spanish Observatorio del Roque de los Muchachos of the Instituto de Astrofisica de Canarias. The data presented here were obtained in part with ALFOSC, which is provided by the Instituto de Astrofisica de Andalucia (IAA) under a joint agreement with the University of Copenhagen and NOTSA. The Liverpool Telescope is operated on the island of La Palma by Liverpool John Moores University in the Spanish Observatorio del Roque de los Muchachos of the Instituto de Astrofisica de Canarias with financial support from the UK Science and Technology Facilities Council. This research is based on observations obtained at the Gemini Observatory under program GN-2010A-Q-30 and principal investigator Edo Berger. Gemini Observatory is operated by the Association of Universities for Research in Astronomy, Inc., under a cooperative agreement with the NSF on behalf of the Gemini partnership: the National Science Foundation (United States), the Science and Technology Facilities Council (United Kingdom), the National Research Council (Canada), CONICYT (Chile), the Australian Research Council (Australia), Ministério da Ciência e Tecnologia (Brazil), and Ministerio de Ciencia, Tecnología e Innovación Productiva (Argentina). We acknowledge the use of public data from the Swift data archive. This research has made use of data obtained through the High Energy Astrophysics Science Archive Research Center Online Service, provided by the NASA/ Goddard Space Flight Center. This research has made use of the XRT Data Analysis Software (XRTDAS) developed under the responsibility of the ASI Science Data Center (ASDC), Italy. The NIST Atomic Spectra Database was used (Ralchenko et al. 2011).

\section{REFERENCES}

Balman, S., Krautter, J., \& Oegelman, H. 1998, ApJ, 499, 395

Berger, E., Soderberg, A. M., Chevalier, R. A., et al. 2009, ApJ, 699, 1850

Bode, M. F., \& Evans, A. (ed.) 2008, Classical Novae (Cambridge: Cambridge Univ. Press)

Botticella, M. T., Pastorello, A., Smartt, S. J., et al. 2009, MNRAS, 398, 1041

Burrows, D. N., Hill, J. E., Nousek, J. A., et al. 2005, SSRv, 120, 165

Capaccioli, M., della Valle, M., Rosino, L., \& D'Onofrio, M. 1989, AJ, 97, 1622

Ciardullo, R., Ford, H. C., Neill, J. D., Jacoby, G. H., \& Shafter, A. W. 1987, ApJ, 318,520

Dalcanton, J. J., Williams, B. F., Seth, A. C., et al. 2009, ApJS, 183, 67

Darnley, M. J., Bode, M. F., Kerins, E., et al. 2006, MNRAS, 369, 257

Della Valle, M. 1991, A\&A, 252, L9

Della Valle, M., \& Duerbeck, H. W. 1993, A\&A, 271, 175

Della Valle, M., Leisy, P., McNaught, R. H., et al. 1991, IAU Circ., 5260, 1

Della Valle, M., \& Livio, M. 1995, ApJ, 452, 704

Della Valle, M., \& Livio, M. 1998, ApJ, 506, 818

della Valle, M., Rosino, L., Bianchini, A., \& Livio, M. 1994, A\&A, 287, 403

Djupvik, A. A., \& Andersen, J. 2010, in Highlights of Spanish Astrophysics V, ed. J. M. Diego et al. (Berlin: Springer-Verlag), 211

Downes, R. A., \& Duerbeck, H. W. 2000, AJ, 120, 2007

Filippenko, A. V. 1997, ARA\&A, 35, 309

Gehrels, N., Chincarini, G., Giommi, P., et al. 2004, ApJ, 611, 1005 
Gilmore, A. C. 1991, IAU Circ., 5253, 3

Gilmore, A. C., Liller, W., Cooper, T., \& Overbeek, D. 1991, IAU Circ., 5250,1

Güver, T., \& Özel, F. 2009, MNRAS, 400, 2050

Hachisu, I., \& Kato, M. 2010, ApJ, 709, 680

Henze, M., Pietsch, W., Haberl, F., et al. 2010, A\&A, 523, A89

Henze, M., Pietsch, W., Haberl, F., et al. 2011, A\&A, 533, A52

Hill, G. J., Nicklas, H. E., MacQueen, P. J., et al. 1998, Proc. SPIE, 3355,375

Hook, I. M., Jørgensen, I., Allington-Smith, J. R., et al. 2004, PASP, 116, 425

Humphreys, R. M., \& Davidson, K. 1994, PASP, 106, 1025

Humphreys, R. M., Prieto, J. L., Rosenfield, P., et al. 2010, ApJL, 718, L43

Iben, J. I., \& Livio, M. 1993, PASP, 105, 1373

Jones, E., Oliphant, T., Peterson, P., et al. 2001, SciPy: Open Source Scientific Tools for Python, http://www.scipy.org

Kasliwal, M. M., Cenko, S. B., Kulkarni, S. R., et al. 2011, ApJ, 735, 94

Landolt, A. U. 1992, AJ, 104, 340

Liller, W., \& Mayer, B. 1987, PASP, 99, 606

Liller, W., McNaught, R. H., Hughes, S. M., et al. 1991, IAU Circ., 5244, 1

Mason, E. 2011, A\&A, 532, L11

McLaughlin, D. B. 1960, in Stellar Atmospheres, ed. J. L. Greenstein (Chicago: Univ. Chicago Press), 585

Nakano, S., \& Kadota, K. 2010, CBET, 2161, 1

Pastorello, A., Botticella, M. T., Trundle, C., et al. 2010, MNRAS, 408, 181

Poole, T. S., Breeveld, A. A., Page, M. J., et al. 2008, MNRAS, 383, 627

Prialnik, D., \& Kovetz, A. 1998, in ASP Conf. Ser. 137, Wild Stars in the Old West, ed. S. Howell, E. Kuulkers, \& C. Woodward (San Francisco, CA: ASP), 376

Prieto, J. L., Kistler, M. D., Thompson, T. A., et al. 2008, ApJL, 681, L9

Ralchenko, Yu., Kramida, A. E., Reader, J., \& NIST ASD Team, 2011, NIST Atomic Spectra Database, ver. 4.1.0, [Online] Available at http://physics.nist.gov/asd3 [2011 August 1] (Gaithersburg, MD: National Institute of Standards and Technology)
Ramsey, L. W., Adams, M. T., Barnes, T. G., et al. 1998, Proc. SPIE, 3352, 34 Roming, P. W. A., Kennedy, T. E., Mason, K. O., et al. 2005, SSRv, 120, 95 Schlegel, D. J., Finkbeiner, D. P., \& Davis, M. 1998, ApJ, 500, 525

Schwarz, G. J., Ness, J.-U., Osborne, J. P., et al. 2011, ApJS, 197, 31 Schwarz, G. J., Shore, S. N., Starrfield, S., et al. 2001, MNRAS, 320, 103 Shafter, A. W. 1997, ApJ, 487, 226

Shafter, A. W., Darnley, M. J., Bode, M. F., \& Ciardullo, R. 2012, ApJ, 752, 156

Shafter, A. W., Darnley, M. J., Hornoch, K., et al. 2011, ApJ, 734, 12

Shafter, A. W., \& Irby, B. K. 2001, ApJ, 563, 749

Shafter, A. W., Rau, A., Quimby, R. M., et al. 2009, ApJ, 690, 1148

Shaviv, N. J. 2001, MNRAS, 326, 126

Shore, S. N., Starrfield, S. G., Sonneborn, G., et al. 1991, IAU Circ., 5257, 1

Smith, N., Li, W., Silverman, J. M., Ganeshalingam, M., \& Filippenko, A. V. 2011, MNRAS, 415, 773

Steele, I. A., Smith, R. J., Rees, P. C., et al. 2004, Proc. SPIE, 5489, 679

Subramaniam, A., \& Anupama, G. C. 2002, A\&A, 390, 449

Szczygieł, D. M., Prieto, J. L., Kochanek, C. S., et al. 2012, ApJ, 750, 77

Thompson, T. A., Prieto, J. L., Stanek, K. Z., et al. 2009, ApJ, 705, 1364

Townsley, D. M., \& Bildsten, L. 2004, ApJ, 600, 390

Townsley, D. M., \& Bildsten, L. 2005, ApJ, 628, 395

Valenti, S., Fraser, M., Benetti, S., et al. 2011, MNRAS, 416, 3138

van den Bergh, S., \& Younger, P. F. 1987, A\&AS, 70, 125

Wagner, R. M., Vrba, F. J., Henden, A. A., et al. 2004, PASP, 116, 326

Warner, B. (ed.) 2003, Cataclysmic Variable Stars (Cambridge: Cambridge Univ. Press)

Williams, R. E. 1990, in IAU Colloq. 122, Physics of Classical Novae, ed. A. Cassatella \& R. Viotti (Lecture Notes in Physics, Vol. 369; Berlin: Springer), 213

Williams, R. E. 1992, AJ, 104, 725

Williams, R. E., Hamuy, M., Phillips, M. M., et al. 1991, ApJ, 376, 721

Williams, R. E., Phillips, M. M., \& Hamuy, M. 1994, ApJS, 90, 297

Yaron, O., Prialnik, D., Shara, M. M., \& Kovetz, A. 2005, ApJ, 623, 398 